TRANSACTIONS OF THE

AMERICAN MATHEMATICAL SOCIETY

Volume 357, Number 8, Pages 3215-3237

S 0002-9947(04)03574-3

Article electronically published on October 7, 2004

\title{
GROUPS OF UNITS OF INTEGRAL GROUP RINGS OF KLEINIAN TYPE
}

\author{
ANTONIO PITA, ÁNGEL DEL RÍO, AND MANUEL RUIZ
}

\begin{abstract}
We explore a method to obtain presentations of the group of units of an integral group ring of some finite groups by using methods on Kleinian groups. We classify the nilpotent finite groups with central commutator for which the method works and apply the method for two concrete groups of order 16
\end{abstract}

\section{INTRODUCTION}

We denote by $R^{*}$ the group of units of a ring $R$ (with identity). Let $G$ be a finite group. The problem of studying the structure of the group of units $\mathbb{Z} G^{*}$ of the integral group ring $\mathbb{Z} G$ has attracted the attention of many authors. The last chapter of the book of Sehgal [17] contains a list of open problems. One of these problems asks for presentations by generators and relations of $\mathbb{Z} G^{*}$ for some finite groups $G$. In this paper we explore a method to obtain presentations of $\mathbb{Z} G^{*}$ for some finite groups $G$ by using techniques on Kleinian groups, that is, discrete subgroups of $\mathrm{PSL}_{2}(\mathbb{C})$. In order to present the main idea it is convenient to consider a more general situation.

Let $A$ be a finite dimensional semisimple rational algebra and $R$ an order in $A$. (By an order, we always mean a $\mathbb{Z}$-order.) It is well known that $R^{*}$ is commensurable with the group of units of every order in $A$ and with $Z(R)^{*} \times R_{1}$, where $R_{1}$ denotes the group of elements of reduced norm 1 of $R$. Recall that two groups $G$ and $H$ are said to be commensurable if there are subgroups $G_{1}$ of $G$ and $H_{1}$ of $H$ such that $\left[G: G_{1}\right]<\infty,\left[H: H_{1}\right]<\infty$ and $G_{1}$ and $H_{1}$ are isomorphic. In particular, if $A=\prod_{x \in X} A_{x}$ where each $A_{x}$ is a simple algebra, then $R^{*}$ is commensurable with $\prod_{x \in X} Z\left(R_{x}\right)^{*} \times \prod_{x \in X}\left(R_{x}\right)_{1}$, where $R_{x}$ is an order in $A_{x}$, for each $x \in X$. Since $Z\left(R_{x}\right)^{*}$ is well understood by the Dirichlet's Unit Theorem, the difficulty in understanding $R^{*}$ up to commensurability relies on understanding the groups of elements of reduced norm 1 of orders in the simple components of the Wedderburn decomposition of $A$. If each simple component $S$ of $A$ can be embedded in $M_{2}(\mathbb{C})$ so that the image of $\left(R_{S}\right)_{1}$ is a discrete subgroup of $\mathrm{SL}_{2}(\mathbb{C})$, for $R_{S}$ an order of $S$, then one can describe $R^{*}$ up to commensurability by using methods on Kleinian groups to describe the groups of $\left(R_{S}\right)_{1}$, for $S$ running on the simple algebras of the Wedderburn decomposition of $A$. In case $A=\mathbb{Q} G$, the rational group algebra of a finite group $G$, then this method could be used to study the group of units of

Received by the editors July 25, 2003 and, in revised form, November 17, 2003.

2000 Mathematics Subject Classification. Primary 16U60; Secondary 11R27, 16S34, 20C05.

This work was partially supported by D.G.I. of Spain and Fundación Séneca of Murcia. 
the integral group ring $\mathbb{Z} G$ which is an order in $\mathbb{Q} G$. This is the motivation of the following definition.

Definition 1.1. A finite dimensional semisimple rational algebra $A$ is said to be of Kleinian type if for every simple quotient $S$ of $A$ there is an embedding $\phi: S \rightarrow$ $M_{2}(\mathbb{C})$ such that $\phi\left(R_{1}\right)$ is a discrete subgroup of $\mathrm{SL}_{2}(\mathbb{C})$ for some order $R$ of $S$.

A finite group $G$ is said to be of Kleinian type if and only if the rational group algebra $\mathbb{Q} G$ is of Kleinian type.

Recall that a presentation of a Kleinian group can be derived from a fundamental polyhedron by using methods that go back to Poincaré and Bianchi 15, 2. Thus if $G$ is a finite group of Kleinian type, then in principle one can obtain a presentation of a group commensurable with $\mathbb{Z} G^{*}$ as follows: first, by computing the Wedderburn decomposition $\prod_{i=1}^{n} A_{i}$ of the rational group algebra $\mathbb{Q} G$ and an order $R_{i}$ of $A_{i}$ for each $i$; second, by applying Dirichlet's Unit Theorem to obtain presentations of $\mathbb{Z}\left(R_{i}\right)^{*}$; third, by computing a fundamental polyhedron of $\left(R_{i}\right)_{1}$ for every $i$; fourth, by using these fundamental polyhedrons to derive presentations of $\left(R_{i}\right)_{1}$ for each $i$ and finally putting all the information together, namely $\mathbb{Z} G^{*}$ is commensurable with a direct product of the groups for which presentations have been obtained.

Once we have explained the main idea we are led to several problems in the hope of obtaining concrete presentations of $\mathbb{Z} G^{*}$ for many groups. The first problem is to understand what is the scope of the method, that is, to classify the finite groups of Kleinian type. The second, but not easier, problem is to obtain fundamental polyhedrons for the discrete groups appearing throughout the process. In this paper we deal with these two problems, but most of the space is devoted to the first one. Notice that if $G$ is of Kleinian type, then all the reduced degrees of $G$ are at most 2. This implies that if $G$ is not nilpotent, then $G$ has a nilpotent subgroup of index $2[6$. This suggests that we concentrate first on the classification of the nilpotent groups of Kleinian type.

Now we explain the contents of the rest of the paper. In Section 2 we establish the basic notation and explain Poincaré's method for obtaining a presentation of a Kleinian group from a fundamental polyhedron. In Section 3 we first characterize the simple algebras of Kleinian type. (Notice that $A$ is of Kleinian type if and only if every simple quotient of $A$ is of Kleinian type.) Then we use this characterization and results from [8] to characterize the finite nilpotent groups of Kleinian type in terms of the Wedderburn decomposition of the corresponding rational group algebra. Using this characterization we obtain the complete list of the finite nilpotent groups of Kleinian type that are not 2-groups. Another consequence of the characterization of the finite nilpotent groups of Kleinian type in terms of its Wedderburn decomposition is that every nonabelian finite nilpotent group of Kleinian type is of the form $G_{1} \times A$ where $G_{1}$ is an indecomposable 2-group (of Kleinian type) and $A$ is an abelian group of exponent a divisor of 4 or 6 . Thus the difficulty of describing the finite nilpotent groups of Kleinian type relies on the 2-groups. In Section 4 we describe explicitly the 2-groups of Kleinian type that satisfy the additional condition that every commutator is central. We finish the paper by obtaining, in Section 5, presentations of a normal complement of $\pm G$ in $\mathbb{Z} G^{*}$, for two concrete finite groups of Kleinian type of order 16. There are nine nonabelian groups of order sixteen: $Q_{8} \times C_{2}, D_{4} \times C_{2}, D_{8}, Q_{16}, D_{16}^{+}, D_{16}^{-}, \mathcal{D}, P$ and $H$ (see Section 2 for the notation). The only one that is not of Kleinian type is $D_{8}$. It is well known that all the units of $\mathbb{Z}\left(Q_{8} \times C_{2}\right)$ are trivial. A description of $\mathbb{Z} G^{*}$ in terms 
of matrices has been obtained for the remaining eight groups in [7] and [10]. If $G=D_{4} \times C_{2}, Q_{16}, P$ or $H$, then $\mathbb{Z} G^{*}$ has a subgroup of finite index which is a direct product of free groups. Such a subgroup with minimal index in $\mathbb{Z} G^{*}$ was explicitly computed in [16] (see also [7, 13]). We obtain presentations for normal complements of the trivial units in $\mathbb{Z} \mathcal{D}^{*}$ and $\left(\mathbb{Z} D_{16}^{+}\right)^{*}$. Since $D_{16}^{-}$is also of Kleinian type, the method explained above is available for this group but the computations seem to be much more complicated than for $\mathcal{D}$ and $D_{16}^{+}$.

\section{Preliminaries}

The group of units of an arbitrary $\operatorname{ring} B$ is denoted by $B^{*}$ and if $B$ is embedded in a finite dimensional rational algebra, then $B_{1}$ denotes the subgroup of $B^{*}$ formed by the elements of reduce norm 1 .

For every positive integer $n, C_{n}$ denotes the cyclic group of order $n, D_{n}$ the dihedral group of order $2 n$ and $Q_{4 n}$ the quaternion group of order $4 n$, that is, $D_{n}$ and $Q_{4 n}$ are given by the following presentations:

$$
\begin{aligned}
& D_{n}=\left\langle a, b \mid a^{n}=b^{2}=1, b a=a^{-1} b\right\rangle, \\
& Q_{4 n}=\left\langle a, b \mid a^{2 n}=a^{n} b^{2}=1, b a=a^{-1} b\right\rangle .
\end{aligned}
$$

We also need the following groups:

$$
\begin{aligned}
& D_{16}^{+}=\left\langle a, b \mid a^{8}=a^{2}=1, b a=a^{5} b\right\rangle \\
& D_{16}^{-}=\left\langle a, b \mid a^{8}=a^{2}=1, b a=a^{3} b\right\rangle \\
& \mathcal{D}=\left\langle a, b, c \mid a^{2}=1=b^{2}, c^{4}=1, a c=c a, b c=c b, b a=c^{2} a b\right\rangle \\
& P=\left\langle a, b \mid a^{4}=b^{4}=1, b a=a^{3} b\right\rangle \\
& H=\left\langle a, b \mid a^{4}=b^{4}=(a b)^{2}=\left[a^{2}, b\right]=1\right\rangle \\
& \mathcal{D}^{+}=\left\langle a, b, c \mid a^{4}=b^{2}=c^{4}, a c=c a, b c=c b, b a=c a^{3} b\right\rangle
\end{aligned}
$$

If $R$ is a commutative ring and $a$ and $b$ are two nonzero elements of $R$, then $\left(\frac{a, b}{R}\right)$ denotes the quaternion $R$-algebra defined by $a$ and $b$, that is, the $R$-algebra given by the following presentation:

$$
\left(\frac{a, b}{R}\right)=R\left[i, j \mid i^{2}=a, j^{2}=b, j i=-i j\right] .
$$

In case $a=b=-1$, then the previous ring is denoted by $\mathbb{H}(R)$.

If $G$ is a group and $X$ is a finite subset of $G$, then $\widehat{X}$ denotes the element of $\mathbb{Q} G$ given by $\widehat{X}=\frac{1}{|X|} \sum_{x \in X} x$. Notice that if $X$ is a subgroup of $G$, then $\widehat{X}$ is idempotent which is central in $\mathbb{Q} G$ if and only if $X$ is normal in $G$.

We finish this section by recalling some basic facts on groups acting on 3dimensional hyperbolic space. We refer to [1, 3] and 4. We are going to use Poincaré's model of the 3-dimensional hyperbolic space, that is, the upper halfspace $H^{3}=\left\{(x, y, r) \in \mathbb{R}^{3}: r>0\right\}$. The projective special linear group $\mathrm{PSL}_{2}(\mathbb{C})$ can be identified with the group $\operatorname{Isom}^{+}\left(H^{3}\right)$ of orientation preserving isometries of $H^{3}$. Recall that a subgroup of $\mathrm{SL}_{2}(\mathbb{C})$ is discrete (in the obvious Euclidean topology) if and only if its image in $\mathrm{PSL}_{2}(\mathbb{C})$ acts discontinuously on $H^{3}$. Such groups are known as Kleinian groups.

If $G$ is a Kleinian group and $D$ is a fundamental polyhedron of $G$, then a presentation of $G$ can be derived from $D$. This is explained in [1] for Fuchsian groups and in 4 and 12 for Fuchsian and Kleinian groups. For the convenience of the reader we explain how to obtain a presentation of $G$ from a fundamental polyhedron $D$ of $G$. The main ingredients of the method are the sides and the edges of $D$. The sides 
are the subsets of $H^{3}$ of the form $s_{g}=\bar{D} \cap g(\bar{D})$ with $g \in G$ which have dimension 2 (that is, contained in an hyperplane of $H^{3}$ but not in a line) and the edges are the sets of the form $e_{g, h}=s_{g} \cap s_{h}$ of dimension 1 (that is contained in a line but not in a point). The sides are congruent in pairs under $G$ (namely if $s_{g}$ is a side, then $s_{g^{-1}}$ is another side and $g\left(s_{g^{-1}}\right)=s_{g}$ ), and the side pairing transformations generate $G$. Let $X \subseteq G$ such that $\left\{s_{g}: g \in X\right\}$ is a set of representatives of the pairs of sides. A full set of relations of $G$ is formed by the reflection relations and the cycle relations. One side $s_{g}$ is paired with itself if and only if $g^{2}=1$. These are the reflection relations. A cycle is a list of even length

$$
\left[e_{1}, g_{1}, e_{2}, g_{2}, e_{3}, \ldots, e_{n}, g_{n}\right]
$$

where for each $i=1, \ldots, n, e_{i}$ is an edge, $g_{i} \in X$ or $g_{i}^{-1} \in X$, and $g_{i}\left(e_{i}\right)=e_{i+1}$ where $e_{n+1}=e_{1}$. Each cycle gives rise to a relation $\left(g_{n} \cdots g_{1}\right)^{k}=1$ where $k$ is the order of $g_{n} \cdots g_{1}$. These are the cycle relations. The value of $k$ can be also computed using geometrical information [1, 4, 12.

\section{Finite groups of Kleinian type}

In this section we first classify the simple algebras of Kleinian type. Note that a finite dimensional semisimple rational algebra is of Kleinian type if and only if its simple quotients are of Kleinian type. Then we use the classification of the simple algebras of Kleinian type to obtain a precise characterization of the finite nilpotent groups of Kleinian type in terms of the Wedderburn decomposition of the corresponding rational group algebra. Finally we use this characterization to describe explicitly all the finite nilpotent groups of Kleinian type that are not 2groups.

Notice that the condition on an order $R$ of a simple finite dimensional rational algebra $S$ in Definition 1.1 does not depend on the particular order selected (see 4, Theorem 2.2.6]). The clue for the proof of the next proposition, that is, the use of the Strong Approximation Theorem, was suggested by Fritz Grunewald in a private communication.

Proposition 3.1. A finite dimensional rational simple algebra $S$ is of Kleinian type if and only if one of the following conditions hold:

(1) $S$ is a number field,

(2) $S$ is a totally definite quaternion algebra,

(3) $S$ is a quaternion algebra over the rationals,

(4) $S=M_{2}(\mathbb{Q}(\sqrt{d}))$ where $d$ is a negative square free integer, or

(5) $S$ is a division quaternion algebra over a number field $K$ with exactly one pair of complex embeddings such that $S$ is ramified at all the real embeddings of $K$.

Proof. If $S$ satisfies condition (1) or (2), then $R_{1}$ is finite for every order in $S$ and so it is of Kleinian type. Obviously $S$ is of Kleinian type if it satisfies condition (3) or (4). Finally, if $S$ satisfies condition (5), then it is of Kleinian type by [3] Theorem 10.1.2].

Conversely, assume that $S$ is of Kleinian type. Since $S$ embeds in $M_{2}(\mathbb{C})$, then necessarily $S$ is either a number field, and therefore condition (1) holds, or $S$ is a quaternion algebra over its centre. In the remainder of the proof we assume that $S$ is a quaternion algebra over its centre $K$. 
Let $\sigma_{1}, \ldots, \sigma_{n}$ be representatives up to conjugation of the nonreal embeddings of $K$ in $\mathbb{C}, \tau_{1}, \ldots, \tau_{k}$ the real embeddings of $K$ at which $S$ does not ramify and $\mu_{1}, \ldots, \mu_{m}$ the real embeddings of $R$ at which $S$ ramifies. Then there are natural embeddings $f_{\sigma_{i}}: S \hookrightarrow M_{2}(\mathbb{C}), f_{\tau_{i}}: S \hookrightarrow M_{2}(\mathbb{R})$ and $f_{\mu_{i}}: S \hookrightarrow \mathbb{H}(\mathbb{R})$. By hypothesis $f_{\rho}\left(R_{1}\right)$ is a discrete subgroup of $\mathrm{SL}_{2}(\mathbb{C})$ for some embedding $\rho: K \rightarrow \mathbb{C}$ and we may assume that $\rho$ is either $\sigma_{n}, \tau_{k}$ or $\mu_{m}$.

Assume first that $\rho=\tau_{k}$. Then $f_{\tau_{k}}\left(R_{1}\right)$ is a Fuchsian group and therefore every free abelian subgroup of $f_{\tau_{k}}\left(R_{1}\right)$ is cyclic. This implies that $K=\mathbb{Q}$, because $f_{\tau_{k}}\left(R_{1}\right)$ contains a lattice of dimension $[K: \mathbb{Q}]$ formed by the elements of the form $\left(\begin{array}{cc}1 & \tau_{k}(x) \\ 0 & 1\end{array}\right)$ with $x$ in the centre of $R$. Thus condition (3) holds.

Assume now that $\rho=\sigma_{n}$ or $\rho=\mu_{m}$. By the Strong Approximation Theorem (see [14] or [18]), if $k \geq 1$, then $f_{\sigma_{1}} \times \cdots \times f_{\sigma_{n}} \times f_{\tau_{2}} \times \cdots \times f_{\tau_{k}} \times f_{\mu_{1}} \times \cdots \times f_{\mu_{m}}$ maps $R_{1}$ into a dense subgroup of $\mathrm{SL}_{2}(\mathbb{C})^{n} \times S L_{2}(\mathbb{R})^{k-1} \times \mathbb{H}(\mathbb{R})_{1}^{m}$ and if $n \geq 2$, then $f_{\sigma_{2}} \times \cdots \times f_{\sigma_{n}} \times f_{\tau_{1}} \times \cdots \times f_{\tau_{k}} \times f_{\mu_{1}} \times \cdots \times f_{\mu_{m}}$ maps $R_{1}$ into a dense subgroup of $\mathrm{SL}_{2}(\mathbb{C})^{n-1} \times S L_{2}(\mathbb{R})^{k} \times \mathbb{H}(\mathbb{R})_{1}^{m}$. The first statement and the hypothesis implies that $k=0$. The second one implies that $n \leq 1$ and if $\rho=\tau_{m}$, then $n=0$. Thus, if $\rho=\mu_{m}$, then condition (2) holds. It only remains to consider the case $\rho=\sigma_{1}$. If $S$ is not a division algebra, then $m=0$ and hence condition (4) holds. Otherwise condition (5) holds.

The following lemma is a direct consequence of the obvious fact that the class of algebras of Kleinian type is closed under quotients and subalgebras.

Lemma 3.2. The class of finite groups of Kleinian type is closed under subgroups and quotients.

Of course Proposition 3.1 provides a characterization of when a finite group $G$ is of Kleinian type in terms of the Wedderburn decomposition of $\mathbb{Q} G$. The following theorem is more precise and provides the simple algebras that occur as quotients of the rational group algebras of groups of Kleinian type.

Theorem 3.3. The following conditions are equivalent for a finite nilpotent group $G$ :

(a) $G$ is of Kleinian type.

(b) Every noncommutative simple quotient of $\mathbb{Q} G$ is isomorphic to either $\mathbb{H}(K)$, with $K=\mathbb{Q}$ or $\mathbb{Q}(\sqrt{2})$, or $M_{2}(K)$, with $K=\mathbb{Q}, \mathbb{Q}(\sqrt{-1}), \mathbb{Q}(\sqrt{-2})$ or $\mathbb{Q}(\sqrt{-3})$.

In this case if e is a primitive central idempotent of $\mathbb{Q} G$, then one of the following cases hold:

(1) If $\mathbb{Q} G e \cong \mathbb{H}(\mathbb{Q})$, then $G e \cong Q_{8}$.

(2) If $\mathbb{Q} G e \cong \mathbb{H}(\mathbb{Q}(\sqrt{2}))$, then $G e \cong Q_{16}$.

(3) If $\mathbb{Q} G e \cong M_{2}(\mathbb{Q})$, then $G e \cong D_{4}$.

(4) If $\mathbb{Q} G e \cong M_{2}(\mathbb{Q}(\sqrt{-1}))$, then $G e \cong D_{16}^{+}$, $\mathcal{D}$ or $\mathcal{D}^{+}$.

(5) If $\mathbb{Q} G e \cong M_{2}(\mathbb{Q}(\sqrt{-2}))$, then $G e \cong D_{16}^{-}$.

(6) If $\mathbb{Q} G e \cong M_{2}(\mathbb{Q}(\sqrt{-3}))$, then $G e \cong D_{4} \times C_{3}$ or $G e \cong Q_{8} \times C_{3}$.

Proof. (b) implies (a) is a direct consequence of Proposition 3.1

Let $G$ be a finite nilpotent group of Kleinian type and $e$ a primitive central idempotent of $\mathbb{Q} G$ such that $\mathbb{Q} G e$ is not commutative. Notice that $G e$ is of Kleinian type 
by Lemma 3.2 Assume first that $\mathbb{Q} G e$ is not a division ring. Then by Proposition 3.1, $\mathbb{Q} G e \cong M_{2}(\mathbb{Q}(\sqrt{d}))$ for $d$ a nonpositive integer. By Theorem [8, Theorem 2.2], $G e$ and $\mathbb{Q} G e$ satisfy one of the conditions (3)-(6).

Assume now that $\mathbb{Q} G e$ is a division ring. Then, by [8, Theorem 2.3], either $G e \cong Q_{2^{n}}$ for $n \geq 3$ or $G e \cong Q_{8} \times C_{n}$ for an odd integer $n>1$ such that the multiplicative order of 2 module $n$ is odd. In the latter case $\mathbb{H}\left(\mathbb{Q}\left(\xi_{n}\right)\right)$ is isomorphic to a simple quotient of $\mathbb{Q} G e$. By Proposition $3.1 \mathbb{Q}\left(\xi_{n}\right)$ has at most one pair of complex embeddings. Thus $\phi(n) \leq 2$, where $\phi$ denotes the Euler function. Since $n$ is odd, this implies that $n=3$, contradicting the fact that the order of 2 module $n$ should be odd. Therefore $G e \cong Q_{2^{n}}$ for $n \geq 3$. Let $K=\mathbb{Q}\left(\xi_{2^{n-2}}+\xi_{2^{n-2}}^{-1}\right)$. Since one of the simple quotients of $Q_{2^{n}}$ is isomorphic to $M_{2}(K)$ and $K$ is totally real, then $K=\mathbb{Q}$, by Proposition 3.1. Thus $n=3$ or 4 . We conclude that $G e \cong Q_{8}$ and $\mathbb{Q} G e \cong \mathbb{H}(\mathbb{Q})$ or $G e=Q_{16}$ and $\mathbb{Q} G e \cong \mathbb{H}(\mathbb{Q}(\sqrt{2}))$.

We close this section with two corollaries of Theorem 3.3 on the structure of the finite nilpotent groups of Kleinian type. The first one shows that the difficulty relies on the identification of the 2-groups of Kleinian type and the second lists explicitly the nilpotent groups of Kleinian type that are not 2-groups.

Corollary 3.4. Let $G$ be a a finite nonabelian nilpotent group of Kleinian type. Then $G=G_{1} \times A$ where $G_{1}$ is an indecomposable nonabelian 2-group of Kleinian type and $A$ is abelian of exponent a divisor of 4 or 6 .

Proof. If $G_{1}$ and $G_{2}$ are two nilpotent nonabelian groups such that $G_{1} \times G_{2}$ is of Kleinian type, then, by Theorem [3.3, $\mathbb{Q}\left(G_{1} \times G_{2}\right) \cong \mathbb{Q} G_{1} \otimes_{\mathbb{Q}} \mathbb{Q} G_{2}$ has a simple quotient in common with one of the following algebras:

$$
\begin{array}{ll}
\mathbb{H}(\mathbb{Q}) \otimes_{\mathbb{Q}} \mathbb{H}(\mathbb{Q}) & \cong \mathbb{H}(\mathbb{Q}) \otimes_{\mathbb{Q}} M_{2}(\mathbb{Q}) \cong M_{2}(\mathbb{H}(\mathbb{Q})), \\
\mathbb{H}(\mathbb{Q}) \otimes_{\mathbb{Q}} \mathbb{H}(\mathbb{Q}(\sqrt{2})) & \cong M_{2}(\mathbb{H}(\mathbb{Q}(\sqrt{2})), \\
\mathbb{H}(\mathbb{Q}) \otimes_{\mathbb{Q}} M_{2}(\mathbb{Q}(\sqrt{-n})) & \cong M_{2}(\mathbb{Q}) \otimes_{\mathbb{Q}} M_{2}\left(\mathbb{Q}(\sqrt{-n}) \cong M_{4}(Q(\sqrt{-n})),\right. \\
M_{2}(\mathbb{Q}(\sqrt{-n})) \otimes_{\mathbb{Q}} M_{2}(\mathbb{Q}(\sqrt{-n})) & \cong 2 M_{4}(\mathbb{Q}(\sqrt{-n})) \\
M_{2}(\mathbb{Q}(\sqrt{-n})) \otimes_{\mathbb{Q}} M_{2}(\mathbb{Q}(\sqrt{-m})) & \cong M_{4}(\mathbb{Q}(\sqrt{-n}, \sqrt{-m}))
\end{array}
$$

for $n$ and $m$ equal to either 1,2 or 3 and $n \neq m$. This contradicts Theorem 3.3

Therefore, if $G$ is a nilpotent nonabelian group of Kleinian type, then $G=$ $G_{1} \times A$ with $G_{1}$ an indecomposable nonabelian group and $A$ an abelian group. Furthermore, by Theorem 3.3, the order of $G_{1}$ is even. If $n$ divides the exponent of $A$, then $G$ has a subgroup $H=G_{1} \times C_{n}$, which is of Kleinian type by Lemma 3.2. Then $\mathbb{Q} H$ has a noncommutative simple quotient $S$ such that $Z(S)$ contains $\mathbb{Q}\left(\xi_{n}\right)$, where $\xi_{n}$ is a primitive $n$-th root of unity. By Theorem 3.3. $\phi(n) \leq 2$ and hence $n$ is a divisor of 4 or 6 .

Corollary 3.5. Let $G$ be a finite nilpotent group which is not a 2-group. Then $G$ is of Kleinian type if and only if $G$ is either abelian or isomorphic to $H \times A$ with $A$ an abelian group of exponent 3 or 6 and $H$ one of the groups given by the following presentations:

(a) $\left\langle x, y \mid x^{4}=y^{4}=\left[x^{2}, y\right]=\left[x, y^{2}\right]=[x,[x, y]]=[y,[x, y]]=1\right\rangle$,

(b) $\left\langle x, y_{1}, \ldots, y_{n} \mid x^{4}=y_{i}^{2}=\left[y_{i}, y_{j}\right]=\left[x^{2}, y_{i}\right]=\left[\left[x, y_{i}\right], y_{j}\right]=\left[\left[x, y_{i}\right], x\right]=1\right\rangle$,

(c) $\left\langle x, y_{1}, \ldots, y_{n} \mid x^{4}=y_{i}^{4}=y_{i}^{2}\left[x, y_{i}\right]=\left[y_{i}, y_{j}\right]=\left[x^{2}, y_{i}\right]=\left[y_{i}^{2}, x\right]=1\right\rangle$,

(d) $\left\langle x, y_{1}, \ldots, y_{n} \mid x^{2}=y_{i}^{2}=\left[y_{i}, y_{j}\right]=\left[\left[x, y_{i}\right], y_{j}\right]=\left[x, y_{i}\right]^{2}=1\right\rangle$,

(e) $\left\langle x, y_{1}, \ldots, y_{n} \mid x^{2}=y_{i}^{4}=y_{i}^{2}\left[x, y_{i}\right]=\left[y_{i}, y_{j}\right]=\left[\left[x, y_{i}\right], x\right]=1\right\rangle$,

(f) $\left\langle x, y_{1}, \ldots, y_{n} \mid x^{4}=y_{i}^{4}=x^{2} y_{1}^{2}=y_{i}^{2}\left[x, y_{i}\right]=\left[y_{i}, y_{j}\right]=\left[y_{i}^{2}, x\right]=1\right\rangle$. 
Proof. By [9], the following conditions are equivalent for a finite nilpotent group $G$ :

(1) Every noncommutative simple quotient of $\mathbb{Q} G$ is isomorphic to either $M_{2}(\mathbb{Q})$ or $\mathbb{H}(\mathbb{Q})$.

(2) $G$ is either abelian or isomorphic to $H \times B$ with $B$ an elementary abelian 2 -group and $H$ one of the groups given by the presentations (a)-(f). (Warning: The list in 9] is displayed in a different way.)

Assume that $G=H \times A$ where $H$ is one of the groups (a)-(f) and $A$ is an abelian group of exponent 3 or 6 . Set $A=B \times C$ where $B$ is an elementary abelian 2-group and $C$ a nontrivial elementary abelian 3 -group. By the previous paragraph, every noncommutative simple component of $\mathbb{Q}(H \times B)$ is isomorphic to either $\mathbb{H}(\mathbb{Q})$ or $M_{2}(\mathbb{Q})$. Since the simple quotients of $\mathbb{Q} A$ are all isomorphic to either $\mathbb{Q}$ or $\mathbb{Q}(\sqrt{-3})$, every noncommutative simple quotient of $\mathbb{Q} G$ is isomorphic to either $\mathbb{H}(\mathbb{Q}), M_{2}(\mathbb{Q})$ or $\mathbb{H}(\mathbb{Q}) \otimes \mathbb{Q}(\sqrt{-3}) \cong M_{2}(\mathbb{Q}(\sqrt{-3})) \cong M_{2}(\mathbb{Q}) \otimes \mathbb{Q}(\sqrt{-3})$. By Theorem 3.3, $G$ is of Kleinian type.

Conversely, assume that $G$ is a nonabelian nilpotent group of Kleinian type which is not a 2-group. By Corollary [3.4 $G \cong H \times B \times C$ where $B$ is an elementary abelian 2-group, $C$ is a nontrivial elementary abelian 3-group and $H$ is an indecomposable 2-group of Kleinian type. If $\mathbb{H}(\mathbb{Q}(\sqrt{2})), M_{2}(\mathbb{Q}(i))$ or $M_{2}(\mathbb{Q}(\sqrt{-2}))$ is isomorphic to a quotient of $\mathbb{Q} H$, then $\mathbb{Q} G$ has a simple quotient isomorphic to either $\mathbb{H}(\mathbb{Q}(\sqrt{2})) \otimes_{\mathbb{Q}} \mathbb{Q}(\sqrt{-3}) \cong M_{2}(\mathbb{Q}(\sqrt{2}, \sqrt{-3}))$ or $M_{2}(\mathbb{Q}(i)) \otimes_{\mathbb{Q}} \mathbb{Q}(\sqrt{-3}) \cong$ $M_{2}(\mathbb{Q}(\sqrt{3}, i))$ or $M_{2}(\mathbb{Q}(\sqrt{-2})) \otimes_{\mathbb{Q}} \mathbb{Q}(\sqrt{-3}) \cong M_{2}(\mathbb{Q}(\sqrt{-2}, \sqrt{-3}))$, contradicting Theorem 3.3. Thus, by Theorem 3.3, every simple quotient of $\mathbb{Q} H$ is isomorphic to either $\mathbb{H}(\mathbb{Q})$ or $M_{2}(\mathbb{Q})$. By the first paragraph of this proof and the indecomposability of $H$ one can deduce that $H$ is isomorphic to one of the groups (a)-(f).

\section{2-GROUPS WITH CENTRAL COMMUTATOR}

In the previous section we have characterized the finite nilpotent groups of Kleinian type in terms of its Wedderburn decomposition. Using this characterization we have obtained a complete description of those which are not 2-groups and have shown that in order to describe all the finite nilpotent groups of Kleinian type it is enough to describe those that are 2-groups. In this section we deal with this problem. Unfortunately we have not been able to obtain a full description of these groups. Nevertheless, we are going to describe all the 2-groups of Kleinian type for which the centre contains the commutator. Namely we prove the following theorem.

Theorem 4.1. Let $G$ be a finite nonabelian 2-group such that $G^{\prime} \subseteq Z(G)$. Then $G$ is of Kleinian type if and only if $G$ is isomorphic to a quotient of $H \times C_{4}^{m}$ where $m \geq 0$ and $H$ is the group given by one of the following presentations:

$$
\begin{aligned}
B_{2}= & \left\langle x_{1}, x_{2} \mid x_{i}^{8}=\left[x_{i}, x_{j}^{4}\right]=\left[x_{i},\left[x_{j}, x_{k}\right]\right]=1, i, j, k=1,2\right\rangle, \\
A_{31}= & \left\langle x_{1}, x_{2}, x_{3} \mid x_{i}^{4}=\left[x_{i}, x_{j}^{2}\right]=\left[x_{i},\left[x_{j}, x_{k}\right]\right]=1,1 \leq i, j, k \leq 3\right\rangle, \\
A_{32}= & \left\langle x_{1}, x_{2}, x_{3} \mid x_{1}^{4}=x_{2}^{4}\left[x_{1}, x_{2}\right]=x_{3}^{4}\left[x_{1}, x_{3}\right]=\left[x_{i}, x_{j}^{2}\right]=1,1 \leq i, j, k \leq 3\right\rangle, \\
B_{n 1}= & \left\langle x_{1}, x_{2}, \ldots, x_{n}\right| x_{1}^{8}=x_{k}^{4}=\left[x_{i}, x_{j}^{2}\right]=\left[x_{k}, x_{l}\right]=\left[x_{i},\left[x_{1}, x_{k}\right]\right]=1, \\
& 1 \leq i, j \leq n, 2 \leq k, l \leq n\rangle,
\end{aligned}
$$




$$
\begin{gathered}
B_{n 2}=\left\langle x_{1}, x_{2}, \ldots, x_{n}\right| x_{1}^{8}=x_{k}^{4}\left[x_{1}, x_{k}\right]=\left[x_{i}, x_{j}^{2}\right]=\left[x_{k}, x_{l}\right]=\left[x_{i},\left[x_{1}, x_{k}\right]\right]=1 \\
1 \leq i, j \leq n, 2 \leq k, l \leq n\rangle .
\end{gathered}
$$

We denote by $\mathcal{G}$ the class of finite 2-groups of Kleinian type $G$ such that $G^{\prime} \subseteq$ $Z(G)$.

The following two lemmas are easy consequences of Lemma 3.2 and Theorem 3.3 .

Lemma 4.2. The class $\mathcal{G}$ is closed under subgroups and homomorphic images.

Lemma 4.3. If $G \in \mathcal{G}$ and $e$ is a primitive central idempotent of $\mathbb{Q} G$, such that $\mathbb{Q} G e$ is noncommutative, then one of the following cases hold:

(1) $\mathbb{Q} G e \cong \mathbb{H}(\mathbb{Q})$ and $G e \cong Q_{8}$.

(2) $\mathbb{Q} G e \cong M_{2}(\mathbb{Q})$ and $G e \cong D_{4}$.

(3) $\mathbb{Q} G e \cong M_{2}(\mathbb{Q}(i))$ and $G e \cong D_{16}^{+}$or $\mathcal{D}$.

Therefore both $(G e)^{\prime}$ and $G e / Z(G e)$ are elementary abelian 2-groups.

Lemma 4.4. If $G \in \mathcal{G}$, then $G \times C_{4} \in \mathcal{G}$.

Proof. The noncommutative simple quotients of $\mathbb{Q} G$ are of the form $\mathbb{H}(\mathbb{Q}), M_{2}(\mathbb{Q})$ and $M_{2}(\mathbb{Q}(i))$, and therefore the noncommutative simple quotients of $\mathbb{Q}\left(G \times C_{4}\right)$ are also of this form.

The following lemma provides a first approach to the description of the elements of $\mathcal{G}$.

Lemma 4.5. If $G$ is a nonabelian element of $\mathcal{G}$, then $Z(G)$ has exponent a divisor of 4 and both $G / Z(G)$ and $G^{\prime}$ are elementary abelian 2-groups.

Proof. Let $e_{1}, e_{2}, \ldots, e_{n}$ be the primitive central idempotent of $\mathbb{Q} G$. Each $G e_{i}$ is a multiplicative subgroup of $\mathbb{Q} G e_{i}$ and the map $f: G \rightarrow \prod_{i=1}^{n} G e_{i}$ defined by $f(g)=\left(g e_{1}, g e_{2}, \ldots, g e_{n}\right)$ is an injective homomorphism of groups such that the composition of $f$ with the projection on each component is surjective.

Assume that $G e_{1}, \ldots, G e_{k}$ are abelian and $G e_{k+1}, \ldots, G e_{n}$ are not abelian. By Lemma 4.3, for each $i>k,\left(G e_{i}\right)^{\prime}$ and $G e_{i} / Z\left(G e_{i}\right)$ are elementary abelian 2 groups. The first implies that the exponent of $G^{\prime}$ is 2 . Let $H=G e_{1} \times \cdots \times G e_{k}$ and $K=G e_{k+1} \times \cdots \times G e_{n}$. Then $f(Z(G)) \subset H \times Z(K)$ and therefore $f$ induces a homomorphism $f^{\prime}: G / Z(G) \rightarrow \prod_{i=k+1}^{n} K / Z(K)=\prod_{i=k+1}^{n} G e_{i} / Z\left(G e_{i}\right)$. Furthermore, $f^{\prime}$ is injective because the composition of $f$ with the projection on every component is surjective. Thus $G / Z(G)$ is elementary abelian.

Let $g \in Z(G)$. Then $g\left(1-\widehat{G^{\prime}}\right)$ is a central unit of $\mathbb{Q} G\left(1-\widehat{G^{\prime}}\right)$. By Lemma 4.3. $\mathbb{Q} G\left(1-\widehat{G^{\prime}}\right) \cong \mathbb{H}(\mathbb{Q})^{m} \times M_{2}(\mathbb{Q})^{s} \times M_{2}(\mathbb{Q}(i))^{r}$ for some $r, s, m \geq 0$. Since the central periodic units of order a power of 2 of $\mathbb{H}(\mathbb{Q}), M_{2}(\mathbb{Q})$ and $M_{2}(\mathbb{Q}(i))$ have order a divisor of $4, g^{4}\left(1-\widehat{G^{\prime}}\right)=\left(1-\widehat{G^{\prime}}\right)$. As $G$ is nonabelian, we have that $G^{\prime} \neq 1$ and comparing coefficients it follows that $g^{4} \in G^{\prime}$ and in fact $g^{4}=1$. Therefore the order of $g$ is a divisor of 4 .

We are going to call the rank of a finite group $G$, denoted $r(G)$, to the minimum of the cardinalities of the generating subsets of $G$. As a consequence of Lemma 4.5 
if $G$ is a nonabelian element of $\mathcal{G}$ and $r(G)=n$, then $G$ is a quotient of a group of the form

$$
B_{n}=\left\langle x_{1}, x_{2}, \ldots, x_{n} \mid x_{i}^{8}=\left[x_{i}, x_{j}^{2}\right]=\left[x_{i},\left[x_{j}, x_{k}\right]\right]=1,1 \leq i, j, k \leq n\right\rangle .
$$

The following proposition proves that the group $B_{2}$ belongs to $\mathcal{G}$ and therefore by Lemma 4.2, the nonabelian groups in $\mathcal{G}$ of rank 2 are precisely the nonabelian quotients of $B_{2}$.

Lemma 4.6. $B_{2} \in \mathcal{G}$.

Proof. The central primitive idempotents $e$ of $\mathbb{Q} B_{2}\left(1-\widehat{B_{2}^{\prime}}\right)$ are the following:

$$
\begin{aligned}
e_{1} & =\left(1-\widehat{B_{2}^{\prime}}\right) \widehat{x_{1}^{2} x_{2}^{2}}, & & e_{6}=\left(1-\widehat{B_{2}^{\prime}}\right)\left(1-\widehat{x_{1}^{2}}\right) \widehat{x_{1}^{4}}\left(1-\widehat{x_{2}^{4}}\right), \\
e_{2} & =\left(1-\widehat{B_{2}^{\prime}}\right) \widehat{x}_{1}^{2} x_{2}^{4}\left(1-\widehat{x_{2}^{2}}\right), & & e_{7}=\left(1-\widehat{B_{2}^{\prime}}\right)\left(1-\widehat{x_{1}^{4}}\right) x_{2}^{4} x_{2}^{2}, \\
e_{3} & =\left(1-\widehat{B_{2}^{\prime}}\right)\left(1-\widehat{x_{1}^{2}}\right) \widehat{x_{1}^{4} x_{2}^{2}}, & & e_{8}=\left(1-\widehat{B_{2}^{\prime}}\right)\left(1-\widehat{x_{1}^{4}}\right) x_{2}^{4}\left(1-\widehat{x_{2}^{2}}\right), \\
e_{4} & =\left(1-\widehat{B_{2}^{\prime}}\right)\left(1-\widehat{x_{1}^{2}}\right) \widehat{x_{1}^{4}}\left(1-\widehat{x_{2}^{2}}\right) \widehat{x_{2}^{4}}, & & e_{9}=\left(1-\widehat{B_{2}^{\prime}}\right)\left(1-\widehat{x_{1}^{4}}\right)\left(1-\widehat{x_{2}^{4}}\right) x_{1}^{2} x_{2}^{2}, \\
e_{5} & =\left(1-\widehat{B_{2}^{\prime}}\right) \widehat{x_{1}^{2}}\left(1-\widehat{x_{2}^{4}}\right), & & e_{10}=\left(1-\widehat{B_{2}^{\prime}}\right)\left(1-\widehat{x_{1}^{4}}\right)\left(1-\widehat{x_{2}^{4}}\right)\left(1-\widehat{x_{1}^{2} x_{2}^{2}}\right) .
\end{aligned}
$$

The corresponding simple algebra $\mathbb{Q} G e_{i}$ is isomorphic to $M_{2}(\mathbb{Q})$ if $i<4$, isomorphic to $\mathbb{H}(\mathbb{Q})$ if $i=4$ and isomorphic to $M_{2}(\mathbb{Q}(i))$ if $i>4$. The proposition now follows from Theorem 3.3 ,

If $B_{n} \in \mathcal{G}$ were true for every $n$, then the elements of $\mathcal{G}$ would be the groups isomorphic to quotients of the $B_{n}$ 's. Unfortunately $B_{n} \notin \mathcal{G}$ if $n \geq 3$. This is a consequence of the next lemma.

Lemma 4.7. Let $G \in \mathcal{G}$ such that $G=\left\langle x_{1}, x_{2}, \ldots, x_{n}, Z(G)\right\rangle$. Fix $i=1,2, \ldots, n$ and let $T=T_{i}=\left\langle\left[x_{i}, x_{j}\right] \mid j \neq i\right\rangle$. If $G^{\prime} \neq T$, then $x_{i}^{4} \in T$.

Proof. If $x_{i}^{4} \notin T$, then the image of $x_{i}$ in $G / T$ is central element of a nonabelian element of $\mathcal{G}$ whose order does not divide 4 , contradicting Lemma 4.5 .

Notation 4.8. For the rest of the section, each time we consider a nonabelian element $G$ of $\mathcal{G}$ we assume that $G$ is a quotient of $B_{n}$. We are going to frequently abuse the notation by denoting by $x_{i}$ both the generators of $B_{n}$ and its image in $G$. We also denote $t_{i j}=\left[x_{i}, x_{j}\right]$, both in $B_{n}$ and $G$.

By Lemma 4.5, $t_{i j}$ and $x_{i}^{2}$ are central elements for all $i, j$. This implies that $t_{i j}^{2}=1$ and hence $t_{i j}=t_{j i}$.

Frequently we are going to claim that one may assume some relation on the $x_{i}$ 's to hold. In that case we mean that the corresponding assumption is possible after some changing on the $x_{i}$ 's. For example, assume that $G=\left\langle x_{1}, x_{2}, x_{3}\right\rangle$ and $r\left(G^{\prime}\right)=2$. Then we may assume that $t_{23}=1$. Indeed, if $t_{i j}=1$ for some $i \neq j$, then the claim follows after reordering the generators. If $t_{13} t_{23}=1$, then the claim follows after replacing $x_{2}$ by $x_{1} x_{2}$ in the list of generators. A combination of this change of generators with a reordering deals with the cases $t_{12} t_{13}=1$ and $t_{12} t_{23}=1$. Finally if $t_{12} t_{13} t_{23}=1$, then the desired conclusion follows after replacing $x_{2}$ and $x_{3}$ by $x_{1} x_{2}$ and $x_{1} x_{3}$, respectively.

From Lemma 4.7 we deduce additional conditions for the groups of rank 3 in $\mathcal{G}$. 
Lemma 4.9. If $G \in \mathcal{G}$ and $r(G)=3$, then $G$ is a quotient of one of the following five groups:

$$
\begin{array}{lll}
B_{2} \times C_{4}, & B_{31}=B_{3} /\left\langle t_{23}, x_{2}^{4}, x_{3}^{4}\right\rangle, & B_{32}=B_{3} /\left\langle t_{23}, x_{2}^{4} t_{12}, x_{3}^{4} t_{13}\right\rangle, \\
A_{31}=B_{3} /\left\langle x_{1}^{4}, x_{2}^{4}, x_{3}^{4}\right\rangle, & A_{32}=B_{3} /\left\langle x_{1}^{4}, x_{2}^{4} t_{12}, x_{3}^{4} t_{13}\right\rangle .
\end{array}
$$

Proof. If $r\left(G^{\prime}\right)=1$, then we may assume that one of the $x_{i}$ is central, for instance, $x_{3}$, and hence $t_{13}=t_{23}=1$. From Lemma 4.5 we obtain that $x_{3}^{4}=1$ and therefore $G$ is a quotient of $B_{2} \times C_{4}=B_{3} /\left\langle t_{13}, t_{23}, x_{3}^{4}\right\rangle$.

If $r\left(G^{\prime}\right)=2$, then we may assume that $t_{23}=1$ and that $t_{12}, t_{13}$ and $t_{12} t_{13}$ are all different to 1 . By Lemma4.7 $x_{2}^{4} \in\left\langle t_{12}\right\rangle, x_{3}^{4} \in\left\langle t_{13}\right\rangle$ and $\left(x_{2} x_{3}\right)^{4} \in\left\langle t_{12} t_{13}\right\rangle$. This implies that either $x_{2}^{4}=x_{3}^{4}=1$ or $x_{2}^{4}=t_{12}$ and $x_{3}^{4}=t_{13}$, that is to say, $G$ is a quotient of $B_{31}$ or $B_{32}$.

Finally, assume that $r\left(G^{\prime}\right)=3$. Using Lemma 4.7 once more we have that there exist $\alpha_{2}, \alpha_{3}, \beta_{1}, \beta_{3}, \gamma_{1}$ and $\gamma_{2}$ in $\{0,1\}$ such that

$$
x_{1}^{4}=t_{12}^{\alpha_{2}} t_{13}^{\alpha_{3}}, \quad x_{2}^{4}=t_{12}^{\beta_{1}} t_{23}^{\beta_{3}} \quad \text { and } \quad x_{3}^{4}=t_{13}^{\gamma_{1}} t_{23}^{\gamma_{2}} .
$$

Then

$$
\begin{aligned}
& \left(x_{1} x_{2}\right)^{4}=t_{12}^{\alpha_{2}+\beta_{1}} t_{13}^{\alpha_{3}} t_{23}^{\beta_{3}} \in\left\langle t_{12}, t_{13} t_{23}\right\rangle, \\
& \left(x_{1} x_{3}\right)^{4}=t_{12}^{\alpha_{2}} t_{13}^{\alpha_{3}+\gamma_{1}} t_{23}^{\gamma_{2}} \in\left\langle t_{13}, t_{12} t_{23}\right\rangle, \\
& \left(x_{2} x_{3}\right)^{4}=t_{12}^{\beta_{1}} t_{13}^{\gamma_{1}} t_{23}^{\beta_{3}+\gamma_{2}} \in\left\langle t_{23}, t_{12} t_{13}\right\rangle,
\end{aligned}
$$

and this implies that $\alpha_{3}=\beta_{3}, \alpha_{2}=\gamma_{2}$ and $\beta_{1}=\gamma_{1}$. Put $a_{1}=\beta_{1}, a_{2}=\alpha_{2}$, and $a_{3}=\alpha_{3}$ for a more friendly notation. Then

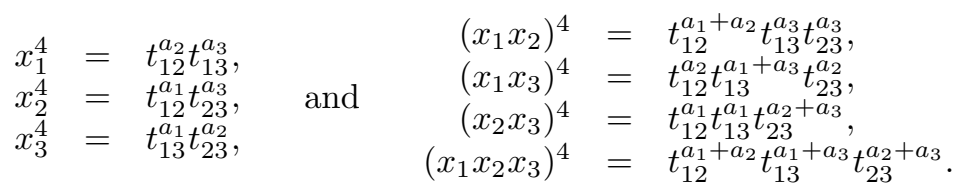

Considering the eight possible values of $\left(a_{1}, a_{2}, a_{3}\right)$, it follows that at least one of the seven elements in (11) is equal to 1 . Thus we may assume that $x_{1}^{4}=1$, and hence $a_{2}=a_{3}=1$. Then $x_{2}^{4}=t_{12}^{a_{1}}$ and $x_{3}^{4}=t_{13}^{a_{1}}$. We conclude that $G$ is a quotient of $A_{31}$ (if $a_{1}=0$ ) or $A_{32}$ (if $a_{1}=1$ ).

In the proof of Theorem 4.1 we will see that the 5 groups of Lemma 4.9 belong to $\mathcal{G}$. This completes the description of the nonabelian elements of $\mathcal{G}$ of rank at most 3 as quotients of 5 groups. To describe the groups of rank greater than 3 the following lemma will be helpful.

Lemma 4.10. If $G \in \mathcal{G}$, then $r(G / Z(G))=2$ if and only if $r\left(G^{\prime}\right)=1$.

Proof. Let $G$ be an arbitrary finite group and $p$ a prime integer. By 11, Lemma 1.4] $G / Z(G) \cong C_{p} \times C_{p}$ if and only if $\left|G^{\prime}\right|=p$ and every nonlinear irreducible complex representation of $G$ has degree $p$. Then the lemma follows noticing that if $G \in \mathcal{G}$, then every irreducible nonlinear complex representation of $G$ has degree 2 .

We are going to consider an elementary abelian 2-group of rank $s$ as an $n$ dimensional vector space over the field $\mathbb{F}_{2}$ with 2 elements. The elements 0 and 1 will be interpreted both as integers and as elements of $\mathbb{F}_{2}$.

Now we are going to introduce some notation. Let $G \in \mathcal{G}$ such that $G^{\prime}=\langle t\rangle \cong$ $C_{2}$. To every list $x_{1}, x_{2}, \ldots, x_{n}$ of elements of $G$ such that $G=\left\langle x_{1}, x_{2}, \ldots, x_{n}, Z(G)\right\rangle$ 
we associate a symmetric matrix $A=\left(\alpha_{i j}\right)_{i j} \in M_{n}\left(\mathbb{F}_{2}\right)$ defined by the formula $t_{i j}=t^{\alpha_{i j}}$.

Consider now the element $x=x_{1}^{\alpha_{1}} \cdots x_{n}^{\alpha_{n}}$ with $\alpha_{i} \in\{0,1\}$. Then $x$ is a central element of $G$ if and only if $\left(\alpha_{1}, \cdots, \alpha_{n}\right)$ belongs to the null space of the matrix $A$. From Lemma 4.10 we obtain that this matrix has rank 2. This trivial observation is going to be used several times without specific mention.

Lemma 4.11. If a finite group $G$ has 4 elements $x_{1}, x_{2}, x_{3}, x_{4}$ such that $\left[x_{1}, x_{2}\right] \neq$ $1,\left[x_{3}, x_{4}\right] \neq 1$ and $\left[x_{i}, x_{j}\right]=1$ for all $1 \leq i \leq 2<j \leq 4$, then $G \notin \mathcal{G}$.

Proof. By Lemma 4.2 we may assume that $G=\left\langle x_{1}, x_{2}, x_{3}, x_{4}\right\rangle$. Then $G^{\prime}=$ $\left\langle\left[x_{1}, x_{2}\right]\right\rangle=\left\langle\left[x_{3}, x_{4}\right]\right\rangle=C_{2}$ and the matrix associated to $x_{1}, x_{2}, x_{3}, x_{4}$ is

$$
\left(\begin{array}{llll}
0 & 1 & 0 & 0 \\
1 & 0 & 0 & 0 \\
0 & 0 & 0 & 1 \\
0 & 0 & 1 & 0
\end{array}\right)
$$

which has rank 4 . Thus $G \notin \mathcal{G}$.

We now prove two lemmas for groups of rank 4 module its centre.

Lemma 4.12. If $G \in \mathcal{G}$ and $r(G / Z(G))=4$, then $r\left(G^{\prime}\right)=3$.

Proof. By Lemma 4.2 we may assume that $r(G)=4$ and therefore $G$ is a quotient of $B_{4}$, say $G=B_{4} / T$. Then $T \subseteq Z\left(B_{4}\right)$ because otherwise $r(G / Z(G)) \leq 3$. Moreover, $G^{\prime} \cong B_{4}^{\prime} /\left(T \cap B_{4}^{\prime}\right)$.

For every permutation $i j k l$ of $\{1,2,3,4\}$ let $M_{i j-k l}=\left\langle t_{i k}, t_{i l}, t_{j k}, t_{j l}, t_{i j} t_{k l}\right\rangle$.

Claim 1: $T \cap B_{4}^{\prime} \nsubseteq M_{i j-k l}$ for any permutation ijkl of $\{1,2,3,4\}$.

We argue by contradiction. By symmetry we may assume that $T \cap B_{4}^{\prime} \subseteq M_{12-34}$. Then $t_{12} \notin T M_{12-34}$ because otherwise we have that $t_{12}=t m$ with $t \in T$ and $m \in M_{12-34}$. Hence $t \in T \cap B_{4}^{\prime} \subseteq M_{12-34}$ and therefore $t_{12} \in M_{12-34}$, a contradiction. Analogously $t_{34} \notin T M_{12-34}$. Then the quotient $H=B_{4} / T M_{12-34}$ verifies the conditions of Lemma 4.11 and therefore $H \notin \mathcal{G}$ which contradicts Lemma 4.2 because $H$ is isomorphic to a quotient of $G$. This proves Claim 1 .

Claim 2: If $r\left(B_{4}^{\prime} \cap T\right) \leq 2$, then $t_{i j} \notin T$ for any $1 \leq i, j \leq 4$ with $i \neq j$.

Claim 2 follows from Claim 1 , if $r\left(T \cap B_{4}^{\prime}\right) \leq 1$. Thus, assume that $r\left(T \cap B_{4}^{\prime}\right)=2$ and that $t_{i j} \in T$ for some $i \neq j$. By symmetry we may assume that $t_{12} \in T$, hence

$$
T \cap B_{4}^{\prime}=\left\langle t_{12}, t_{13}^{\alpha_{1}} t_{14}^{\alpha_{2}} t_{23}^{\alpha_{3}} t_{24}^{\alpha_{4}} t_{34}^{\alpha_{5}}\right\rangle
$$

for some $\alpha_{i} \in\{0,1\}$, not all 0 . Since $T \cap B_{4}^{\prime} \nsubseteq M_{13-24}$ and $T \cap B_{4}^{\prime} \nsubseteq M_{14-23}$ we have that $\alpha_{1} \neq \alpha_{4}$ and $\alpha_{2} \neq \alpha_{3}$. By symmetry one may assume that $\alpha_{1}=1$ and therefore $\alpha_{4}=0$. If $\alpha_{2}=1$, then $T \cap B_{4}^{\prime}=\left\langle t_{12}, t_{13} t_{14} t_{34}^{\alpha_{5}}\right\rangle$. Replacing $x_{3}$ by $x_{3} x_{4}$ we have that $T \cap B_{4}^{\prime}=\left\langle t_{12}, t_{13} t_{34}^{\alpha_{5}}\right\rangle \subseteq M_{14-23}$, a contradiction. Therefore $\alpha_{2}=0$, and hence $T \cap B_{4}^{\prime}=\left\langle t_{12}, t_{13} t_{23} t_{34}^{\alpha_{5}}\right\rangle$. If $\alpha_{5}=0$, replacing $x_{1}$ by $x_{1} x_{2}$ we have that $T \cap B_{4}^{\prime} \subseteq M_{14-23}$ and if $\alpha_{5}=1$ replacing $x_{4}$ by $x_{1} x_{2} x_{4}$ we have that $T \cap B_{4}^{\prime} \subseteq M_{13-24}$ a contradiction. This proves Claim 2.

Claim 3: $r\left(B_{4}^{\prime} \cap T\right) \geq 3$.

Assume that $r\left(B_{4}^{\prime} \cap \bar{T}\right) \leq 2$ and therefore $t_{i j} \notin T$ for all $i \neq j$, by Claim 2 . Then one may assume that $t_{12} t_{13}^{\alpha_{1}} t_{14}^{\alpha_{2}} t_{23}^{\alpha_{3}} t_{24}^{\alpha_{4}} t_{34}^{\alpha_{5}} \in T$ for some $\alpha_{i} \in\{0,1\}$, not all 0 . Replacing $x_{2}$ and $x_{1}$ by $x_{2} x_{3}^{\alpha_{1}} x_{4}^{\alpha_{2}}$ and $x_{1} x_{3}^{\alpha_{3}} x_{4}^{\alpha_{4}}$ respectively we may assume that 
$t_{12} t_{34}^{\alpha_{5}} \in T$. Since $t_{i j} \notin T$, we have that $\alpha_{5}=1$, that is to say, $t_{12} t_{34} \in T$. Therefore there are (new) $\alpha_{i}$ 's such that

$$
T \cap B_{4}^{\prime}=\left\langle t_{12} t_{34}, t=t_{12}^{\alpha_{1}} t_{13}^{\alpha_{2}} t_{14}^{\alpha_{3}} t_{23}^{\alpha_{4}} t_{24}^{\alpha_{5}} t_{34}^{\alpha_{6}}\right\rangle
$$

Since $T \cap B_{4}^{\prime} \not \subset M_{12-34}$, necessarily $\alpha_{1} \neq \alpha_{6}$. By symmetry, we may assume that $\alpha_{1}=1$ and $\alpha_{6}=0$ obtaining that $T \cap B_{4}^{\prime}=\left\langle t_{12} t_{34}, t_{12} t_{13}^{\alpha_{2}} t_{14}^{\alpha_{3}} t_{23}^{\alpha_{4}} t_{24}^{\alpha_{5}}\right\rangle$. Arguing similarly $\alpha_{2} \neq \alpha_{5}$ and $\alpha_{3} \neq \alpha_{4}$. Again taking advantage of the symmetry we may assume that $\alpha_{2}=1$ and therefore $\alpha_{5}=0$. If $\alpha_{3}=1$, then $T \cap B_{4}^{\prime}=\left\langle t_{12} t_{34}, t_{12} t_{13} t_{14}\right\rangle$. Replacing $x_{2}$ by $x_{2} x_{3} x_{4}$ we obtain that $t_{12} \in T$, a contradiction. If $\alpha_{3}=0$, then $T \cap B_{4}^{\prime}=\left\langle t_{12} t_{34}, t_{12} t_{13} t_{23}\right\rangle$. Replacing $x_{1}$ by $x_{1} x_{2} x_{4}$ we obtain that $t_{13} \in T$, again a contradiction. This proves Claim 3 .

As a consequence of Claim 3 and Lemma 4.10 one concludes that $2 \leq r\left(G^{\prime}\right) \leq 3$ and it only remains to prove that $r\left(G^{\prime}\right) \neq 2$. By means of contradiction we assume that $r\left(G^{\prime}\right)=2$.

So far in this proof we have used $t_{i j}$ to denote commutators in $B_{4}$. Now we are going to change the notation and put $G=\left\langle x_{1}, x_{2}, x_{3}, x_{4}\right\rangle$ and adopt the conventions of Notation 4.8.

Claim 4: One may assume that $G^{\prime}=\left\langle t_{12}, t_{13}\right\rangle$.

Proving Claim 4 is equivalent to proving that one may assume that $G^{\prime}=$ $\left\langle t_{a x}, t_{a y}\right\rangle$ for some $a, x, y \in\{1,2,3,4\}$. We may assume that $t_{12} \neq 1$. Let $H=$ $\left\langle t_{12}, t_{13}, t_{14}, t_{23}, t_{24}\right\rangle$. If $r(H)=2$, we may assume that $t_{12}$ and $t_{13}$ are linearly independent. Otherwise we have that $H=\left\langle t_{12}\right\rangle$ and since $r\left(G^{\prime}\right)=2$, we deduce that $G^{\prime}=\left\langle t_{12}, t_{34}\right\rangle$. By Lemma 4.11 there exist $1 \leq i \leq 2<j \leq 4$ such that $t_{i j} \neq 1$. Since $t_{i j} \in H, t_{i j}=t_{12}$ and hence $G^{\prime}=\left\langle t_{i j}, t_{34}\right\rangle$. This finishes the proof of Claim 4.

Therefore one may assume that $G^{\prime}=\left\langle t_{12}, t_{13}\right\rangle$ and so one may assume also that $t_{23}=1$, as explained in Notation 4.8 Let us write $t_{i 4}=t_{12}^{\alpha_{i}} t_{13}^{\beta_{i}}$ with $i=1,2,3$. If $H=G /\left\langle t_{12}\right\rangle$, then $H^{\prime}=t_{13} \cong C_{2}$ and the associated matrix is (see paragraph before Lemma 4.11)

$$
\left(\begin{array}{cccc}
0 & 0 & 1 & \beta_{1} \\
0 & 0 & 0 & \beta_{2} \\
1 & 0 & 0 & \beta_{3} \\
\beta_{1} & \beta_{2} & \beta_{3} & 0
\end{array}\right) .
$$

As the rank of this matrix has to be $2, \beta_{2}=0$. By a similar argument with $H=G /\left\langle t_{13}\right\rangle$ we obtain the associated matrix

$$
\left(\begin{array}{cccc}
0 & 1 & 0 & \alpha_{1} \\
1 & 0 & 0 & \alpha_{2} \\
0 & 0 & 0 & \alpha_{3} \\
\alpha_{1} & \alpha_{2} & \alpha_{3} & 0
\end{array}\right)
$$

from which we deduce that $\alpha_{3}=0$. Consider now $H=G /\left\langle t_{12} t_{13}\right\rangle$, obtaining the associated matrix

$$
\left(\begin{array}{cccc}
0 & 1 & 1 & \alpha_{1}+\beta_{1} \\
1 & 0 & 0 & \alpha_{2} \\
1 & 0 & 0 & \beta_{3} \\
\alpha_{1}+\beta_{1} & \alpha_{2} & \beta_{3} & 0
\end{array}\right)
$$

and therefore $\alpha_{2}=\beta_{3}$. Summarizing,

$$
t_{14}=t_{12}^{\alpha_{1}} t_{13}^{\beta_{1}}, \quad t_{24}=t_{12}^{\alpha_{2}}, \quad t_{34}=t_{13}^{\alpha_{2}} .
$$


Replacing $x_{4}$ by $x_{1}^{\alpha_{2}} x_{4}$ we may assume that $\alpha_{2}=0$. Then $x_{2}^{\alpha_{1}} x_{3}^{\beta_{1}} x_{4}$ is a central element of $G$ contradicting the initial hypothesis. In conclusion $r\left(G^{\prime}\right)=3$, finishing the proof of this lemma.

Lemma 4.13. If $G \in \mathcal{G}$ and $r(G / Z(G))=4$, then there exist four elements $x_{1}, x_{2}, x_{3}, x_{4} \in G$ such that $G=\left\langle x_{1}, x_{2}, x_{3}, x_{4}, Z(G)\right\rangle, G^{\prime}=\left\langle t_{12}, t_{13}, t_{14}\right\rangle \cong C_{2}^{3}$ and $t_{23}=t_{24}=t_{34}=1$.

Proof. By assumption there are $x_{1}, x_{2}, x_{3}, x_{4}$ such that $G=\left\langle x_{1}, x_{2}, x_{3}, x_{4}, Z(G)\right\rangle$. We adopt the conventions of Notation 4.8. First we prove that one may assume that $G^{\prime}=\left\langle t_{12}, t_{13}, t_{14}\right\rangle \cong C_{2}^{3}$. Assume the contrary. Then by Lemma 4.12, $t_{a x}, t_{a y}, t_{a z}$ are linearly dependent for every permutation axyz of $\{1,2,3,4\}$. Since $r\left(G^{\prime}\right)=3$, we may assume that $t_{12}$ and $t_{13}$ are linearly independent.

Assume that $G^{\prime}=\left\langle t_{12}, t_{13}, t_{23}\right\rangle$. Since $t_{a x}, t_{a y}, t_{a z}$ are linearly dependent for every permutation axyz of $\{1,2,3,4\}$, necessarily $t_{14} \in\left\langle t_{12}, t_{13}\right\rangle, t_{24} \in\left\langle t_{12}, t_{23}\right\rangle$ and $t_{34} \in\left\langle t_{13}, t_{23}\right\rangle$. If $t_{14}=t_{12}^{a} t_{13}^{b}$, then by replacing $x_{4}$ by $x_{2}^{a} x_{3}^{b} x_{4}$, we may assume that $t_{14}=1$. Let us write

$$
t_{24}=t_{12}^{\alpha_{1}} t_{23}^{\alpha_{3}} \quad \text { and } \quad t_{34}=t_{13}^{\beta_{1}} t_{23}^{\beta_{2}} .
$$

Arguing as in the proof of Lemma 4.12 using $G /\left\langle t_{12}, t_{13} t_{23}\right\rangle, G /\left\langle t_{13}, t_{12} t_{23}\right\rangle$ and $G /\left\langle t_{23}, t_{12} t_{13}\right\rangle$ we obtain that the three matrices

$$
\begin{gathered}
\left(\begin{array}{cccc}
0 & 0 & 1 & 0 \\
0 & 0 & 1 & \alpha_{3} \\
1 & 1 & 0 & \beta_{1}+\beta_{2} \\
0 & \alpha_{3} & \beta_{1}+\beta_{2} & 0
\end{array}\right), \\
\left(\begin{array}{cccccc}
0 & 1 & 1 & 0 \\
1 & 0 & 0 & \alpha_{1} \\
1 & 0 & 0 & \beta_{1} \\
0 & \alpha_{1} & \beta_{1} & 0
\end{array}\right)
\end{gathered}
$$

have rank 2. Therefore $\alpha_{3}=\beta_{2}=0$ and $\alpha_{1}=\beta_{1}$. This implies that $x_{1}^{\alpha_{1}} x_{4}$ is central, which yields to a contradiction.

We have proved that $G^{\prime} \neq\left\langle t_{12}, t_{13}, t_{23}\right\rangle$ and therefore either $G^{\prime}=\left\langle t_{12}, t_{13}, t_{24}\right\rangle$ or $G^{\prime}=\left\langle t_{12}, t_{13}, t_{34}\right\rangle$. Since $x_{2}$ and $x_{3}$ play symmetric roles, we may assume that $G^{\prime}=\left\langle t_{12}, t_{13}, t_{24}\right\rangle$. Moreover, as we are assuming that $t_{a x}, t_{a y}, t_{a z}$ are linearly dependent for every permutation axyz of $\{1,2,3,4\}$, we have that $t_{23} \in\left\langle t_{12}, t_{13}\right\rangle \cap$ $\left\langle t_{12}, t_{24}\right\rangle=\left\langle t_{12}\right\rangle$. We may assume that $t_{23}=1$, by replacing $x_{3}$ by $x_{1} x_{3}$ if necessary. Besides $t_{14} \in\left\langle t_{12}, t_{13}\right\rangle$, hence $t_{14}=t_{12}^{a} t_{13}^{b}$ and replacing $x_{4}$ by $x_{2}^{a} x_{3}^{b} x_{4}$ we obtain that $t_{14}=1$. The derived subgroup of the groups $G /\left\langle t_{12}, t_{13} t_{24}\right\rangle$ has rank 2 and its associated matrix is

$$
\left(\begin{array}{cccc}
0 & 0 & 1 & 0 \\
0 & 0 & 0 & 1 \\
1 & 0 & 0 & b+c \\
0 & 1 & b+c & 0
\end{array}\right)
$$

which has rank 4, a contradiction.

Therefore we have proved that we may assume that $G^{\prime}=\left\langle t_{12}, t_{13}, t_{14}\right\rangle$. Let us see that we also may assume that $t_{23}=t_{24}=t_{34}=1$. 
If $t_{23} \notin\left\langle t_{12}, t_{13}\right\rangle$, by considering $G /\left\langle t_{12}, t_{13}\right\rangle$, we obtain a matrix of the form

$$
\left(\begin{array}{llll}
0 & 0 & 0 & 1 \\
0 & 0 & 1 & * \\
0 & 1 & 0 & * \\
1 & * & * & 0
\end{array}\right)
$$

which has rank 4 . Therefore $t_{23} \in\left\langle t_{12}, t_{13}\right\rangle$ and by similar arguments we have that $t_{24} \in\left\langle t_{12}, t_{14}\right\rangle$ and $t_{34} \in\left\langle t_{13}, t_{14}\right\rangle$. If $t_{23}=t_{12}^{a} t_{13}^{b}$, replacing $x_{2}$ and $x_{3}$ by $x_{1}^{b} x_{2}$ and $x_{1}^{a} x_{3}$ respectively, we may assume that $t_{23}=1$. Let us write

$$
t_{24}=t_{12}^{a_{1}} t_{14}^{c_{1}}, \quad t_{34}=t_{13}^{b_{2}} t_{14}^{c_{2}}
$$

with $a_{i}, b_{i}, c_{i} \in\{0,1\}$. Replacing $x_{4}$ by $x_{1}^{a_{1}} x_{4}$ we may assume that $a_{1}=0$. By considering the following quotients $G /\left\langle t_{12} t_{14}, t_{13}\right\rangle, G /\left\langle t_{12}, t_{13} t_{14}\right\rangle$ and $G /\left\langle t_{12} t_{13}, t_{14}\right\rangle$, we obtain the three matrices

$$
\left(\begin{array}{cccc}
0 & 1 & 0 & 1 \\
1 & 0 & 0 & c_{1} \\
0 & 0 & 0 & c_{2} \\
1 & c_{1} & c_{2} & 0
\end{array}\right), \quad\left(\begin{array}{cccc}
0 & 0 & 1 & 1 \\
0 & 0 & 0 & c_{1} \\
1 & 0 & 0 & b_{2}+c_{2} \\
1 & c_{1} & b_{2}+c_{2} & 0
\end{array}\right), \quad\left(\begin{array}{cccc}
0 & 1 & 1 & 0 \\
1 & 0 & 0 & 0 \\
1 & 0 & 0 & b_{2} \\
0 & 0 & b_{2} & 0
\end{array}\right)
$$

and deduce that $c_{2}=c_{1}=b_{2}=0$, that is to say, $t_{24}=t_{34}=1$ which finishes the proof.

The next step is generalizing Lemma 4.13 to groups of rank at least 4 . We will argue by induction and for the inductive step we need the following Lemma.

Lemma 4.14. Let $G \in \mathcal{G}$ such that $r(G / Z(G))=n \geq 3$. Then there exist a subgroup $H$ of $G$ such that $r(H / Z(H))=r(G / Z(G))-1$.

Proof. We argue by induction in $n$. The result is trivial for $n=3$. Assume that $n \geq 4$ and the induction hypothesis. Let $H$ be a subgroup of $G$ such that

$$
\begin{aligned}
r(H / Z(H))=\max \{r(K / Z(K)): & K \text { a subgroup of } G \text { such that } \\
& Z(G) \subseteq K \text { and } r(K / Z(K)) \neq n\} .
\end{aligned}
$$

Clearly $Z(G) \subseteq H$ and $2 \leq r(H / Z(H))<n$. Let $\left\{x_{1} Z(H), x_{2} Z(H), \ldots, x_{k} Z(H)\right\}$ be a basis of $H / Z(H)$. Then $\left\{x_{1} Z(G), x_{2} Z(G), \ldots, x_{k} Z(G)\right\}$ are linearly independent. Let $\left\{x_{1} Z(G), x_{2} Z(G), \ldots, x_{n} Z(G)\right\}$ be a basis of $G / Z(G)$. Assume that $k \leq n-2$. For every $k<i \leq n$ let $K_{i}=\left\langle H, x_{i}\right\rangle$. Then $k \leq r\left(K_{i} / Z\left(K_{i}\right)\right) \leq k+1<n$. Thus $r\left(K_{i} / Z\left(K_{i}\right)\right)=k$, by the maximality of $k$, and $H=K_{i}$, by the maximality of $H$. Thus $G=\left\langle x_{1}, \ldots, x_{k}, Z(G)\right\rangle$, which yields to a contradiction. Thus $r(H / Z(H))=n-1$ and the lemma follows.

We can now generalize Lemma 4.13

Lemma 4.15. Let $G \in \mathcal{G}$ such that $r(G / Z(G))=n \geq 4$. Then $r\left(G^{\prime}\right)=n-1$ and there exist $x_{1}, x_{2}, \ldots, x_{n} \in G$ such that $G=\left\langle x_{1}, x_{2}, \ldots, x_{n}, Z(G)\right\rangle, G^{\prime}=$ $\left\langle t_{12}, t_{13}, \ldots, t_{1 n}\right\rangle$ and $t_{i j}=1$ for $2 \leq i<j \leq n$.

Proof. We argue by induction on $n$ with the case $n=4$ being Lemma 4.12 Assume that $n>4$ and the induction hypothesis.

By Lemma 4.14, $G$ has a subgroup $H$ such that $r(H / Z(H))=n-1$. By the induction hypothesis there exist $x_{1}, x_{2}, \ldots, x_{n-1} \in H$ such that $H=\left\langle x_{1}, x_{2}, \ldots, x_{n-1}\right.$, 
$Z(H)\rangle, t_{i j}=1$ for every $2 \leq i \leq n-1$ and $t_{12}, \ldots, t_{1(n-1)}$ are linearly independent. In particular, $r\left(G^{\prime}\right) \geq n-2$. Then there exists an element $x_{n} \in G$ such that $G=\left\langle x_{1}, x_{2}, \ldots, x_{n}, Z(G)\right\rangle$. We adopt the conventions of Notation 4.8 .

Notice that the induction hypothesis implies that if $S \in \mathcal{G}$ with $r(S / Z(S))=n$, then $r\left(S^{\prime}\right) \geq n-2$.

Claim 1: If $r\left(G^{\prime}\right)=n-2$, then $t_{i n} \in\left\langle t_{1 i}\right\rangle$ for every $1<i<n$.

Assume that $r\left(G^{\prime}\right)=n-2$. Then $G^{\prime}=\left\langle t_{12}, \ldots, t_{1(n-1)}\right\rangle$ and hence $t_{1 n}=$ $t_{12}^{a_{2}} \ldots t_{1(n-1)}^{a_{n-1}}$ for some $a_{i} \in\{0,1\}$. We argue by contradiction and taking advantage of the symmetry we may assume that $t_{2 n} \notin\left\langle t_{12}\right\rangle$. Let $S=G /\left\langle t_{12}\right\rangle$ and adopt the conventions of Notation 4.8 in the group $S$. With this notation we have that $t_{13}, \ldots, t_{1(n-1)}$ are linearly independent and $t_{12}=1 \neq t_{2 n}$. Let $y=x_{1}^{a_{1}} \ldots x_{n}^{a_{n}} \in$ $Z(S)$ with $a_{i} \in\{0,1\}$. Then $1=\left[x_{2}, y\right]=t_{2 n}^{a_{n}}$ and therefore $a_{n}=0$. Moreover, $1=\left[x_{1}, y\right]=t_{13}^{a_{3}} \ldots t_{1(n-1)}^{a_{n-1}}$ and since $t_{13}, \ldots, t_{1(n-1)}$ are linearly independent, we have that $a_{3}=\cdots=a_{n-1}=0$. Finally $1=\left[x_{3}, y\right]=t_{13}^{a_{1}}$, hence $a_{1}=0$. This proves that $x_{1} Z(S), \ldots, x_{n} Z(S)$ are linearly independent and therefore $r(S / Z(S))=n$. This yields to a contradiction because $r\left(S^{\prime}\right)=n-3$ and Claim 1 follows.

Claim 2: $r\left(G^{\prime}\right) \geq n-1$.

Otherwise $G^{\prime}=\left\langle t_{12} \ldots t_{1(n-1)}\right\rangle \cong C_{2}^{n-2}$ and hence $t_{1 n}=t_{12}^{a_{2}} \ldots t_{1(n-1)}^{a_{n-1}}$ for some $a_{i} \in\{0,1\}$. Then replacing $x_{n}$ by $x_{2}^{a_{2}} \ldots x_{n-1}^{a_{n-1}} x_{n}$ we may assume that $t_{1 n}=1$. By Claim 1 , for every $1<i<n$, either $t_{i n}=1$ or $t_{i n}=t_{1 i}$. Since $x_{n}$ is not central, there exists a $2 \leq i<n$ such that $t_{i n}=t_{1 i}$. Moreover, as $x_{1} x_{n}$ is not central, there exists $2 \leq i<n$ such that $t_{i n} \neq t_{1 i}$ and therefore $t_{i n}=1$. By reordering the $x_{i}^{\prime} \mathrm{s}$, if necessary, we may assume that $t_{3 n}=t_{13}$ and $t_{2 n}=1$. Now we obtain a contradiction by showing that the group $S=G /\left\langle t_{12} t_{13}\right\rangle$ satisfies $r\left(S^{\prime}\right)=n-3$ and $r(S / Z(S))=n$. The former equality is obvious. To prove the latter equality notice that (adopting Notation 4.8 for the group $S$ ) $t_{12}=t_{13}=t_{3 n} \neq 1$. Let $y=x_{1}^{a_{1}} \ldots x_{n}^{a_{n}} \in Z(S)$ with $a_{i} \in\{0,1\}$. Then $1=\left[x_{2}, y\right]=t_{12}^{a_{1}}$ and hence $a_{1}=0$. Besides $1=\left[x_{3}, y\right]=t_{3 n}^{a_{n}}$, hence $a_{n}=0$. Moreover, $1=\left[x_{1}, y\right]=t_{13}^{a_{2}+a_{3}} t_{14}^{a_{4}} \ldots t_{1(n-1)}^{a_{n-1}}$ and since $t_{13}, \ldots, t_{1(n-1)}$ are linearly independent, we have that $a_{4}=\cdots=a_{n-1}=$ 0 and $a_{2}=a_{3}$. Finally $1=\left[x_{n}, y\right]=t_{3 n}^{a_{3}}$, hence $0=a_{3}=a_{2}$. This finishes the proof of Claim 2.

Claim 3: $r\left(G^{\prime}\right)=n-1$.

Recall that $t_{i j}=1$ for $2 \leq i<j \leq n-1$ and so $G^{\prime}=\left\langle t_{1 i}, t_{i n}, t_{1 n}: 2 \leq i<n\right\rangle$.

Assume that $t_{12}, \ldots, t_{1(n-1)}, t_{1 n}$ are linearly independent. Let $1<i, j<n$ with $i \neq j$. By the case $n=4$ we have that $r\left(\left\langle x_{1}, x_{i}, x_{j}, x_{n}\right\rangle^{\prime}\right) \leq 3$ and therefore $t_{i n} \in\left\langle t_{1 i}, t_{1 j}, t_{1 n}\right\rangle$, thus $G^{\prime}=\left\langle t_{12}, \ldots, t_{1(n-1)}, t_{1 n}\right\rangle$ and hence $r\left(G^{\prime}\right)=n-1$.

Otherwise, that is, if $t_{12}, \ldots, t_{1(n-1)}, t_{1 n}$ are linearly dependent, then there exists $1<i<n$ such that $t_{12}, \ldots, t_{1(n-1)}, t_{i n}$ are linearly independent, by Claim 2 . Let $1<j<n$ with $j \neq i$. By the case $n=4$ we have that $r\left(\left\langle x_{1}, x_{i}, x_{j}, x_{n}\right\rangle^{\prime}\right) \leq 3$ and therefore $t_{1 n}, t_{j n} \in\left\langle t_{1 i}, t_{1 j}, t_{i n}\right\rangle$. Thus $G^{\prime}=\left\langle t_{12}, \ldots, t_{1(n-1)}, t_{i n}\right\rangle$ and hence $r\left(G^{\prime}\right)=n-1$. This finishes the proof of Claim 3 .

Claim 4: $t_{\text {in }} \in\left\langle t_{1 i}\right\rangle$ for every $1<i<n$.

For simplicity take $i=2$. If $t_{2 n} \notin\left\langle t_{12}\right\rangle$, let $S=G /\left\langle t_{12}\right\rangle$. Clearly $r\left(S^{\prime}\right)=n-2$ and proving that $r(S / Z(S))=n$ we obtain a contradiction with Claim 3. Let $y=x_{1}^{a_{1}} \ldots x_{n}^{a_{n}} \in Z(S)$ with $a_{i} \in\{0,1\}$. Then $1=\left[x_{2}, y\right]=t_{12}^{a_{n}}$ and therefore $a_{n}=0$. We also have that $1=\left[x_{3}, y\right]=t_{13}^{a_{1}}$, hence $a_{1}=0$. Besides $1=\left[x_{1}, y\right]=$ $t_{13}^{a_{3}} \ldots t_{1(n-1)}^{a_{n-1}}$, hence $a_{3}=\cdots=a_{n-1}=0$. Finally $1=\left[x_{n}, y\right]=t_{2 n}^{a_{2}}$ thus $a_{2}=0$. Thus Claim 4 follows. 
To finish the proof consider the quotient $S=G /\left\langle t_{1 i} t_{1 j}\right\rangle$ for $1<i<j<n$. Arguing as before we show that the assumptions $t_{i n}=1$ and $t_{1 j}=t_{n j}$ imply that $r(S / Z(S))=n$ and $r\left(S^{\prime}\right)=n-2$ yielding a contradiction with Claim 3 . Thus $t_{\text {in }}=1$ for all $1<i<n$ or $t_{i n}=t_{1 i}$ for all $1<i<n$. The second case reduces to the first one by changing $x_{1}$ by $x_{1} x_{n}$ and this finishes the proof of this lemma.

Now we are ready to prove Theorem 4.1 .

Proof of Theorem 4.1. We start by proving that if $G$ is a quotient of $H \times C_{4}^{m}$ with $m \geq 0$ and $H=B_{2}, A_{31}, A_{32}, B_{n 1}$ or $B_{n 2}$ with $n \geq 3$, then $G \in \mathcal{G}$. By Lemmas 4.2 and 4.4 it is enough to show that $H \in \mathcal{G}$ for the five possible values of $H$. We have already seen in Lemma 4.6 that $B_{2} \in \mathcal{G}$. So let $G=A_{31}, A_{32}, B_{n 1}$ or $B_{n 2}$, with $n \geq 3$.

Let $\mathcal{H}$ be the set of maximal subgroups of $G^{\prime}$. We claim that it is enough to show that $G / S \in \mathcal{G}$ for every $S \in \mathcal{H}$. Indeed, if $X$ is a noncommutative simple quotient of $\mathbb{Q} G$, then $X$ is a quotient of $\mathbb{Q} G\left(1-\widehat{G^{\prime}}\right)$. Since $\left\{\widehat{S}\left(1-\widehat{G^{\prime}}\right) \mid S \in \mathcal{H}\right\}$ is a complete set of orthogonal central idempotents of $\mathbb{Q} G\left(1-\widehat{G^{\prime}}\right), X$ is a $\mathbb{Q} G \widehat{S}\left(1-\widehat{G^{\prime}}\right)$ for some $S \in \mathcal{H}$, and hence $X$ is a quotient of $\mathbb{Q} G \widehat{S}$. Since $\mathbb{Q} G \widehat{S} \cong \mathbb{Q}(G / S), X$ is isomorphic to a simple quotient of $\mathbb{Q}(G / S)$. Thus, if $G / S \in \mathcal{G}$, then $X$ is of Kleinian type and so $G \in \mathcal{G}$.

So fix $S \in \mathcal{H}$ and prove that $G / S \in \mathcal{G}$.

Suppose first that $G=A_{31}$ or $A_{32}$. Then $S$ is of one of the following forms: $\left\langle t_{i j}, t_{i k}\right\rangle,\left\langle t_{i j}, t_{i k} t_{j k}\right\rangle,\left\langle t_{12} t_{13}, t_{13} t_{23}\right\rangle$. Since $Z(G / S)$ strictly contains $Z(G) / S$, then $r((G / S) / Z(G / S))=2$. Let $x, y \in G / S$ be two elements linearly independent modulo $Z(G / S)$. Then $G / S=\langle x, y\rangle \times C_{4}$ where $\langle x, y\rangle$ is isomorphic to a quotient of $B_{2}$ and therefore $G / S \in \mathcal{G}$, by Lemma 4.4

Suppose now that $G=B_{n 1}$ or $B_{n 2}$. Consider the map $\varphi: G^{\prime} \rightarrow G$ given by $\varphi\left(t_{12}^{a_{2}} \cdots t_{1 n}^{a_{n}}\right)=x_{2}^{a_{2}} \cdots x_{n}^{a_{n}}$ for $\left(a_{2}, \ldots, a_{n}\right) \in\{0,1\}^{n-1}$. Notice that $\left[x_{1}, \varphi(x)\right]=x$, for every $x \in G^{\prime}$. Let $\left\{c_{3}, \ldots, c_{n}\right\}$ be a basis of $S$ and $\left\{c_{2}, c_{3}, \ldots, c_{n}\right\}$ a basis of $G^{\prime}$. Let $y_{i}=\varphi\left(c_{i}\right)$. Then $\left\{x_{1} Z(G), y_{2} Z(G), \ldots, y_{n} Z(G)\right\}$ is a basis for $G / Z(G)$ and therefore $G=\left\langle x_{1}, y_{2}, \ldots, y_{n}\right\rangle$. We may assume, without loss of generality, that $y_{i}=x_{i}$, that is to say, $\left[x_{1}, x_{i}\right] \in S$ for $i \geq 3$ and $\left[x_{1}, x_{2}\right] \in G^{\prime} \backslash S$. Therefore

$$
G / S \cong\left\langle x_{1}, x_{2}\right\rangle \times\left\langle x_{3}, \ldots, x_{n}\right\rangle \cong\left\langle x_{1}, x_{2}\right\rangle \times C_{4}^{n-2}
$$

and $\left\langle x_{1}, x_{2}\right\rangle$ is isomorphic to a quotient of $B_{2}$. By Lemma $4.4 G / S \in \mathcal{G}$ and this finishes the first part of the proof.

Conversely, let $G$ be a nonabelian element of $\mathcal{G}$ and set $n=r(G / Z(G))$. Select elements $x_{1}, x_{2}, \ldots, x_{n}$ of $G$ such that $\left\{x_{1} Z(G), x_{2} Z(G), \ldots, x_{n} Z(G)\right\}$ is a basis of $G / Z(G)$. Let $Z(G)=\left\langle z_{1}\right\rangle \times \cdots \times\left\langle z_{m}\right\rangle$ with $z_{i}$ of order $k_{i}$. If $H=\left\langle x_{1}, x_{2}, \ldots, x_{n}\right\rangle$, then $H$ has a presentation of the form $\left\langle x_{1}, x_{2}, \ldots, x_{n} \mid R\right\rangle$ for some set of relations $R$. Moreover,

$$
Z(G)=\left\langle z_{1}, \ldots, z_{m} \mid z_{i}^{k_{i}}=\left[z_{i}, z_{j}\right]=1,1 \leq i, j \leq m\right\rangle
$$

is a presentation of $Z(G)$. Then $G$ has a presentation of the form

$$
\left\langle x_{1}, \ldots, x_{n}, z_{1}, \ldots, z_{m} \mid R, z_{i}^{k_{i}}=\left[z_{i}, z_{j}\right]=\left[z_{i}, x_{k}\right]=1, h=z,(h, z) \in T\right\rangle
$$

where $T=\{(h, z) \in H \times Z(G): h=z$, in $G\}$. By Lemma 4.5, $k_{i}$ divides 4 for each $i=1,2, \ldots, m$ and therefore $G$ is isomorphic to a quotient of

$$
\left\langle x_{1}, \ldots, x_{n}, z_{1}, \ldots, z_{m} \mid R, z_{i}^{4}=\left[z_{i}, z_{j}\right]=\left[z_{i}, x_{k}\right]=1\right\rangle=H \times C_{4}^{m} .
$$


Since $r(H)=R(H / Z(H))=n$, we have proved that we may assume without loss of generality that $n=r(G)=r(G / Z(G))$.

Since $G$ is nonabelian, $n \geq 2$. If $n=2$, then $G$ is a quotient of $B_{2}$. If $n=3$, then by Lemma 4.9, $G$ is a quotient of $B_{2} \times C_{4}, A_{31}, A_{32}, B_{31}$ or $B_{32}$. Nevertheless, from the hypothesis $r(G / Z(G))=3$ we deduce that the first case does not hold. Finally assume that $n \geq 4$. By Lemma 4.15, $r\left(G^{\prime}\right)=n-1$ and we may choose the $x_{i}$ 's such that $G^{\prime}=\left\langle t_{12}, \ldots, t_{1 n}\right\rangle$ and $t_{i j}=1$ for $2 \leq i, j \leq n$. The relations $x_{i}^{8}=\left[x_{i}, x_{j}^{2}\right]=\left[x_{i}, t_{i j}\right]=1$ are deduced from Lemma 4.5. By Lemma 4.7 we have that $x_{i}^{4} \in\left\langle t_{1 i}\right\rangle$ for all $2 \leq i \leq n$. Then we only have to prove that either $x_{i}^{4}=1$ for all $2 \leq i \leq n$ or $x_{i}^{4}=t_{1 i}$ for all $2 \leq i \leq n$. Otherwise, we may assume that $x_{2}^{4}=1$ and $x_{3}^{4}=t_{13}$. However, in this case $\left(x_{2} x_{3}\right)^{4}=t_{13} \notin\left\langle\left[x_{1}, x_{2} x_{3}\right]\right\rangle$ which contradicts Lemma 4.7.

\section{TWO EXAMPLES}

In this section we are going to show how to use methods on Kleinian groups to obtain presentations of the group of units of $\mathbb{Z D}$ and $\mathbb{Z} D_{16}^{+}$. We present the method with all the details for $\mathcal{D}$ and avoid the technical details for $D_{16}^{+}$.

By [7] $\mathbb{Z} \mathcal{D}^{*}=G \rtimes \pm \mathcal{D}$ where $G$ is the subgroup of $\mathrm{PSL}_{2}(\mathbb{Z}[i])$ represented by the matrices of the form

$$
I+2\left(\begin{array}{cc}
\alpha & 2 \beta \\
\gamma & \delta
\end{array}\right)
$$

with $\alpha, \beta, \gamma, \delta \in \mathbb{Z}[i]$. Moreover, the action of $\mathcal{D}$ on $G$ can be described by identifying $\mathcal{D}$ with a subgroup of $\mathrm{GL}_{2}(\mathbb{Z}[i])$ via the following identifications:

$$
a=\left(\begin{array}{cc}
1 & 2 \\
0 & -1
\end{array}\right), b=\left(\begin{array}{cc}
-1 & 0 \\
1 & 1
\end{array}\right), c=i I .
$$

Thus to obtain a presentation of $\mathbb{Z} \mathcal{D}^{*}$ it only remains to produce a presentation of the group $G$. In order to do that we use Poincaré's method (see Section 2), so we need to obtain a fundamental polyhedron of $G$. We use Poincaré's model $H^{3}$ of the 3-dimensional hyperbolic space and follow the method explained in Chapter 7 of [3], which is a variation of the Ford Method [5]. Namely if $F_{\infty}$ is a fundamental polyhedron of the stabilizer $G_{\infty}$ of $\infty$, then the intersection of $F_{\infty}$ with the outside part of the isometric half spheres of $G \backslash G_{\infty}$ is a fundamental polyhedron of $G$. Recall that $g=\left(\begin{array}{ll}a & b \\ c & d\end{array}\right) \in \mathrm{SL}_{2}(\mathbb{C})$ stabilizes $\infty$ if and only if $c=0$. If $g$ does not stabilize $\infty$, then the isometric circle of $g$ is the circle of $\mathbb{C}=\mathbb{R}^{2}$ given by $|c z+d|=1$, that is the circle centred at $-d / c$ of radius $1 /|c|$, and the isometric half sphere of $g$ is the intersection with $H^{3}$ of the sphere of $\mathbb{R}^{3}$ having the same centre and radius as the isometric circle of $g$, where $\mathbb{C}$ is identified with the boundary of $H^{3}$ in the obvious way. Since

$$
G_{\infty}=\left\langle\left(\begin{array}{cc}
1 & 4 \\
0 & 1
\end{array}\right),\left(\begin{array}{cc}
1 & 4 i \\
0 & 1
\end{array}\right)\right\rangle,
$$

a fundamental polyhedron of $G_{\infty}$ is the infinite strip $F_{\infty}=[-2,2]^{2} \times \mathbb{R}^{+}$. The radius of the isometric spheres of the elements of $G \backslash G_{\infty}$ are of the form $1 / 2|\gamma|$ for $0 \neq \gamma \in \mathbb{Z}[i]$. Therefore the maximum value taken by this radius is $1 / 2$ and this maximum radius is reached by the elements of the form (2) with $\gamma= \pm 1$ or $\pm i$. The centres of these isometric spheres are the elements of $C=(1 / 2+\mathbb{Z}[i]) \cup(i / 2+\mathbb{Z}[i])$. 
Let $F_{1}$ be the subset of $H^{3}$ formed by the external to the spheres of radius $1 / 2$ with centre in $C$. Consider the lattice $L$ of $\mathbb{Q}[i]$ generated by $\frac{1+i}{2}$ and $\frac{1-i}{2}$. It is not difficult to see that each element of $L$ belongs to the border of exactly four of the spheres that form the border of $F_{1}$ and extending the geodesics of $H^{3}$ to its border (that is $\mathbb{C} \cup\{\infty\}$ ) and abusing slightly of the notation one can describe $F_{1}$ as the hull of $L$ in $H^{3}$. Using this fact, now we can see that the isometric spheres of the elements of $G$ of the form (2) with $\gamma \neq 1$ do not intersect the interior of $F_{1}$ and therefore $F=F_{\infty} \cap F_{1}$ is the searched fundamental polyhedron of $G$. Indeed, the isometric circle of an element as in (2) with $\gamma \neq 0$ is given by the equation $|2 \gamma z+(1+2 \delta)|=1$. If this circle intersects the interior of $F_{1}$, then it contains an element $z \in L$ because $F_{1}$ is the hull of $L$. Thus $|1+2(\gamma z+\delta)|<1$. Let $x, y \in \mathbb{Z}$ such that $z=x \frac{1+i}{2}-y \frac{1-i}{2}=(x+y i) \frac{1+i}{2}$. Then $|1+(\gamma(x+y i)(1+i)+2 \delta)|<1$. Therefore $-1=\gamma(x+y i)(1+i)+2 \delta \in \mathbb{Z}[i](1+i)$, a contradiction. Resuming, we get

Proposition 5.1. The set

$$
\begin{aligned}
& F=\left\{P=z+r j=(x, y, r) \in H^{3}:|x|,|y| \leq 2,|1+2(\gamma z+\delta)|^{2}+r^{2} \mid<1,\right. \\
&\gamma=1 \text { or } i, \delta \in \mathbb{Z}[i]\}
\end{aligned}
$$

is a fundamental polyhedron of the subgroup of $\mathrm{PSL}_{2}(\mathbb{Z}[i])$ formed by the elements represented by the matrices of the form (2) with $\alpha, \beta, \gamma, \delta \in \mathbb{Z}[i]$.

The boundary of the fundamental polyhedron $F$ is formed by parts of forty-four geodesic planes of $H^{3}$ (four half planes and forty half spheres), called sides. Figure 1 represents $F$ and the orthogonal projection of $F$ on the plane $z=0$, where the four sides embedded in half planes project into the four external sides of the square and the forty sides embedded in half spheres project into either squares or triangles. Each side is of the form $s_{g}=F \cap g^{-1}(F)$ for some $g \in G$. The bold diagonals in Figure 1 emphasize the fact that $F$ is invariant under a rotation of $\pi / 2$ degrees around the line $z=0$. This rotation can be realized by the action of the matrix

$$
\alpha=\left(\begin{array}{cc}
\xi_{8}^{-1} & 0 \\
0 & \xi_{8}
\end{array}\right)
$$

where $\xi_{8}=\sqrt{i}$ is a primitive 8 -th root of unity. The invariance of $F$ with respect to this rotation reflects the fact that $G$ is invariant under conjugation by $\alpha$.

In order to identify the $g$ 's such that $s_{g}$ is a side we introduce some notation. For a $g \in G$ and $i \in \mathbb{Z}$, let us denote $g_{i}=\alpha^{-i} g \alpha^{i}$. Note that if $s_{g}$ is a side, then $s_{g_{i}}$ is another side and can be obtained rotating $s_{g}$ counterclockwise $i \frac{\pi}{2}$ degrees. Then all the sides are of the form $s_{g}$ with $g=M_{i}$ or $g=M_{i}^{-1}$ for $i=0,1,2$ or 3 and $M$ is equal to one of the following elements:

$$
\begin{array}{cc}
A=\left(\begin{array}{cc}
1 & -4 \\
0 & 1
\end{array}\right), \quad B=\left(\begin{array}{cc}
1 & 0 \\
-2 & 1
\end{array}\right), \quad C=\left(\begin{array}{cc}
3 & -4 \\
-2 & 3
\end{array}\right), \\
W=\left(\begin{array}{cc}
3-2 i & -4 \\
-2 i & -1+2 i
\end{array}\right), \quad X=\left(\begin{array}{cc}
-1-2 i & 4 i \\
-2 & 3+2 i
\end{array}\right), \\
Y=\left(\begin{array}{cc}
3+4 i & -12 i \\
2 i & 3-4 i
\end{array}\right), \quad Z=\left(\begin{array}{cc}
1+4 i & -8 i \\
2 i & 1-4 i
\end{array}\right) .
\end{array}
$$



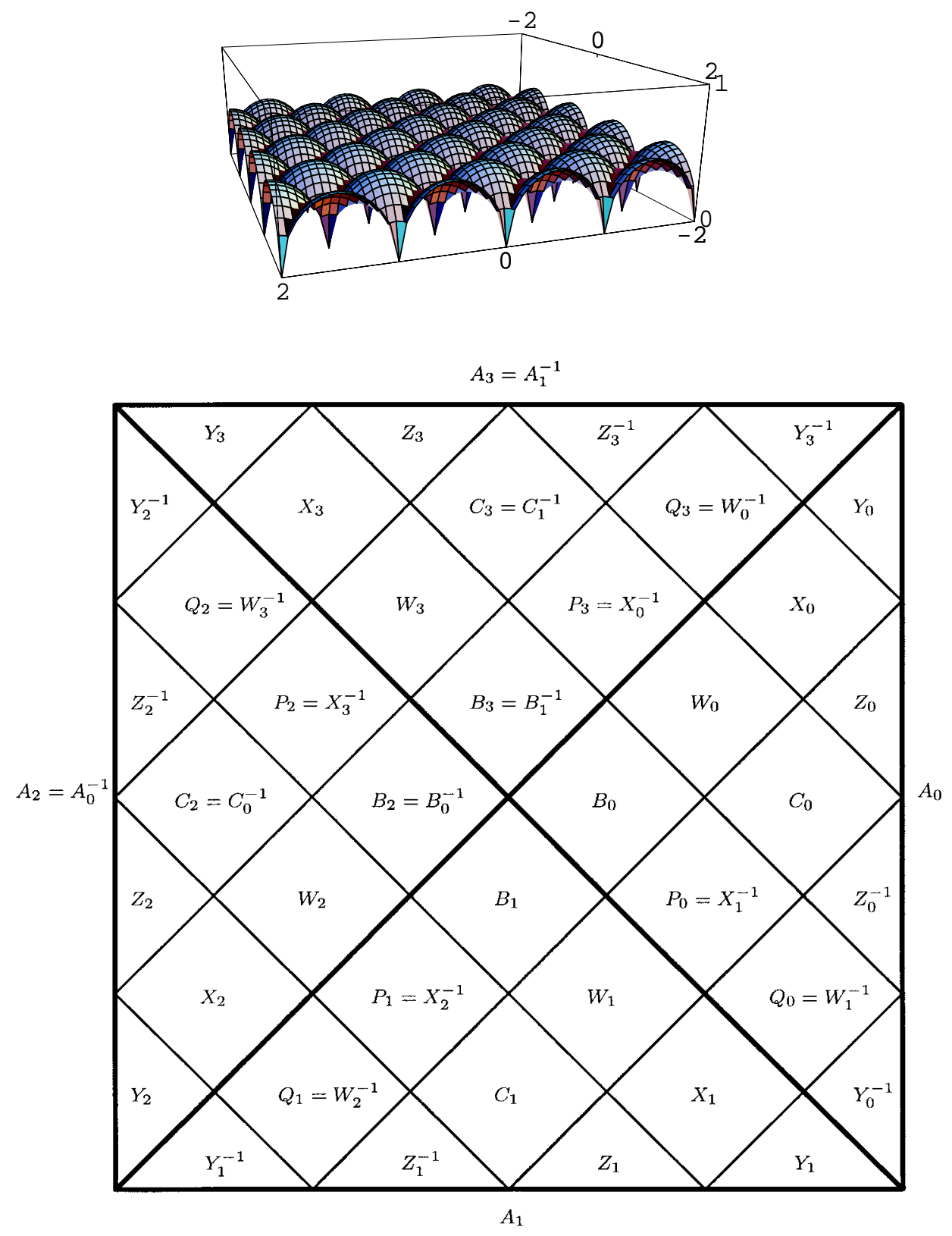

FIGURE 1.

The legend in each side $s$ of the picture above represents the element $g \in G$ such that $s=s_{g}$. Note that the legend in the sides that are planes is displayed "at" rather than "in" the side.

Now a generating set of $G$ readily follows [1]:

$$
G=\left\langle A_{i}, B_{i}, C_{i}, W_{j}, X_{j}, Y_{j}, Z_{j} ; i=0,1 ; j=0,1,2,3\right\rangle .
$$


From now on we call generators to the elements in the previous list of generators of $G$. Since no side is paired with itself there are not reflection relations. In order to produce a presentation of $G$ now we need to compute the cycles of edges of $F$.

The fundamental polyhedron has eighty-four edges, sixty-four edges are the intersection of two isometric spheres, sixteen edges are the intersection of one of the four vertical planes in the border of $F$ with one isometric circle and the remaining four edges are the intersection of two vertical planes. Of course, in principal, one can construct infinitely many cycles. However, some of the relations obtained from cycles can be dropped according to the following three principles. First, clearly cyclic permutations of even order of the cycles give rise to new cycles with equivalent associated relation. Therefore these two cycles are considered as equal. Second, we only need irreducible lists, that is, $g_{i+1}$ should be different than $g_{i}^{-1}$ for each $i$. Finally we only need to consider cycles so that $e_{i} \neq e_{1}$ for $i=2, \ldots, n$. Indeed, a cycle $C$ not satisfying this condition can be obtained by merging two shorter cycles $C_{1}$ and $C_{2}$. It is not difficult to see that the group $G$ is torsion free and therefore the cycle relation associated to $C$ is a consequence of cycle relations associated to $C_{1}$ and $C_{2}$.

Notice that if $e$ is an edge of the side $s_{g}$, then $g(e)$ is an edge of the side $g(s)$. If $f$ is another edge (of any side) such that $g(f)$ is embedded in $F$, then the hull of $g(e)$ and $g(f)$ is embedded in $g(s)=F \cap g(F)$. Hence the closure containing $e$ and $f$ is embedded in the side $s_{g}$ and therefore $f$ is one of the edges of $s_{g}$. In other words, if $g$ is a generator and $f$ is an edge such that $g(f)$ is another edge, then $f$ is one of the edges of precisely $s_{g}$. Thus in a cycle, each $g_{i}$ should be the generators associated to one of two sides containing $e_{i}$.

We are going to explode the symmetry associated to the invariancy by the action of $\alpha$ to classify the cycles in 9 kind of cycles. Figure 2 displays one representative of each kind of cycle. (In the first picture the small circle correspond to the vertical edges.) The first three pictures are invariant under the action of $\alpha$ and hence each picture gives rise to exactly one cycle. The three corresponding relations associated are:

$$
\left[A_{0}, A_{1}\right]=\left[B_{0}, B_{1}\right]=Y_{3} Y_{2} Y_{1} Y_{0}=1 .
$$

The next three pictures are invariant by the action of $\alpha^{2}$ but not by the action of $\alpha$, so that each of them corresponds to two cycles and accordingly to two cycle relations. One of these relations can by obtained by conjugating by $\alpha$ the other relation. The corresponding six relations are:

$$
\begin{aligned}
& C_{0}^{-1} Z_{2} C_{0} Z_{0}=A_{0}^{-1} Z_{2} A_{0} Z_{0}=A_{0}^{-1} Y_{2} A_{0} Y_{0}= \\
& C_{1}^{-1} Z_{3} C_{1} Z_{1}=A_{1}^{-1} Z_{3} A_{1} Z_{1}=A_{1}^{-1} Y_{3} A_{1} Y_{1}=1 .
\end{aligned}
$$

Finally the group generated by $\alpha$ acts faithfully on the last three pictures and therefore each of the last three cycles represents four cycles and so gives rise to four cycle relations. So we have twelve more relations:

$$
\begin{aligned}
& {\left[W_{0}, X_{0}\right]=B_{1} W_{1}^{-1} Z_{0} X_{0}^{-1}=X_{0}^{-1} C_{1} W_{1}^{-1} Y_{0}=} \\
& {\left[W_{1}, X_{1}\right]=B_{0} W_{2}^{-1} Z_{1} X_{1}^{-1}=X_{1}^{-1} C_{0} W_{2}^{-1} Y_{1}=} \\
& {\left[W_{2}, X_{2}\right]=B_{1} W_{3}^{-1} Z_{2} X_{2}^{-1}=X_{2}^{-1} C_{1} W_{3}^{-1} Y_{2}=} \\
& {\left[W_{3}, X_{3}\right]=B_{0} W_{0}^{-1} Z_{3} X_{3}^{-1}=X_{3}^{-1} C_{0} W_{0}^{-1} Y_{3}=1 .}
\end{aligned}
$$



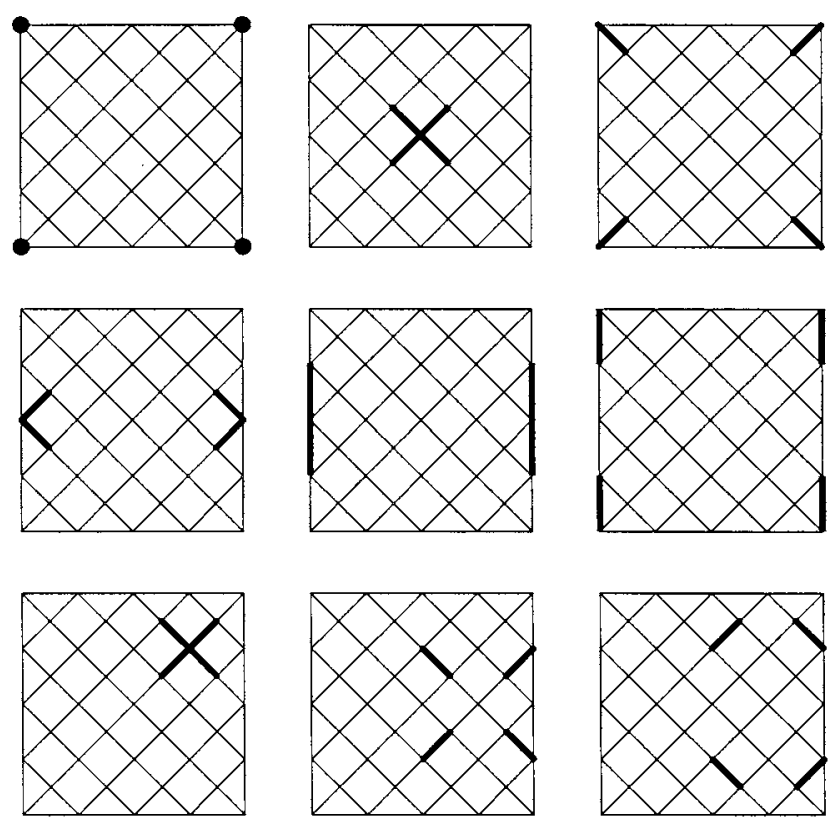

FiguRE 2

We conclude that

Theorem 5.2. The group of trivial units of $\mathbb{Z D}$ for

$$
\left.\mathcal{D}=\langle a, b, c| a^{2}=1=b^{2}, c^{4}=1, b a=c^{2} a b, \text { c central }\right\rangle
$$

has a normal complement in the group of units of $\mathbb{Z D}$ isomorphic to the group given by the following presentation:

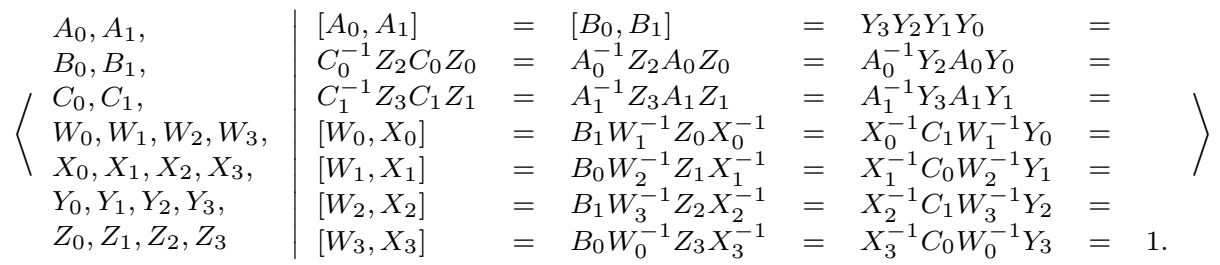

Now we consider the group $D_{16}^{+}$. By [7] $D_{16}^{+}$has a normal complement on the group of units of $\mathbb{Z} D_{16}^{+}$which is isomorphic to the image $G$ in $\operatorname{PSL}_{2}(\mathbb{C})$ of the subgroups of $\mathrm{SL}_{2}(\mathbb{Z}[i])$ given by the matrices of the form

$$
\left(\begin{array}{cc}
1+2 a & 2 b \\
2 c & 1+2 d
\end{array}\right)
$$

with $a, b, c, d \in \mathbb{Z}[i]$ and $b i+c \in 2 \mathbb{Z}[i]$. Using the same method shown in the previous example one shows that $G$ has the same fundamental polyhedron $F$ as in the previous example but the basic generators of the previous example should be 


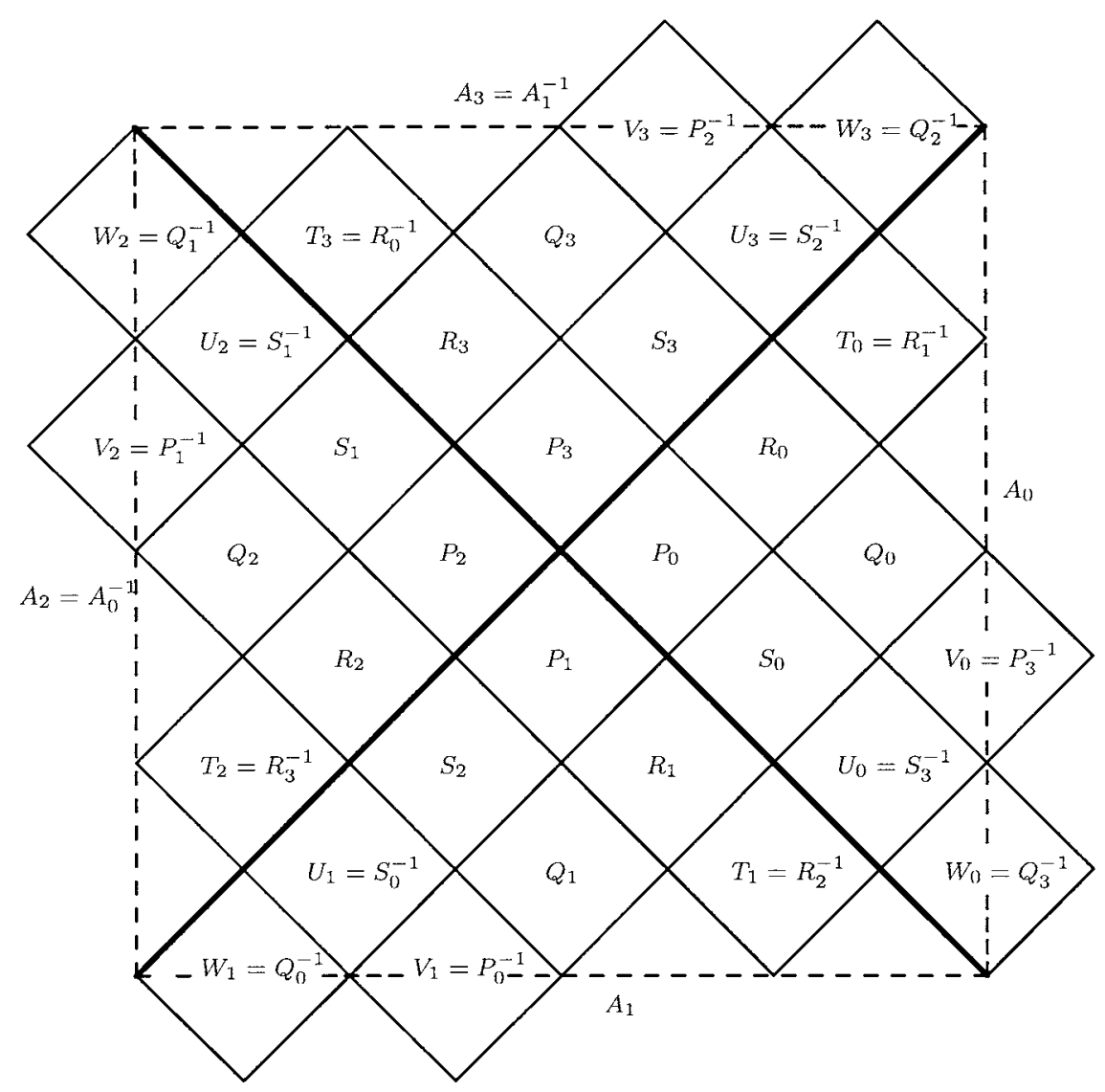

FiguRE 3.

replaced by the following:

$$
\begin{aligned}
& A=\left(\begin{array}{cc}
1 & -4 \\
0 & 1
\end{array}\right), \quad P=\left(\begin{array}{cc}
-1-4 i & 2 i \\
2 & -1
\end{array}\right) \text {, } \\
& Q=\left(\begin{array}{cc}
-3-4 i & 4+6 i \\
2 & -3
\end{array}\right), \quad R=\left(\begin{array}{cc}
-3-2 i & 2+4 i \\
2 i & 1-2 i
\end{array}\right) \text {, } \\
& S=\left(\begin{array}{cc}
3-2 i & -2+4 i \\
2 i & -1-2 i
\end{array}\right) \text {. }
\end{aligned}
$$

In this example it is convenient to change slightly the fundamental polyhedron by "cutting" some of the external triangles of the projection of $F$ and replace them by the image of these triangles by the action of either $A_{0}$ or $A_{1}$. Then the projection of the fundamental polyhedron in Figure 1 now takes the form of the picture of Figure 3 . Using the same method as in the previous example one obtains the following.

Theorem 5.3. The group of trivial units of $\mathbb{Z} D_{16}^{+}$for

$$
D_{16}^{+}=\left\langle a, b \mid a^{8}=1=b^{2}, b a=a^{5} b\right\rangle
$$


has a normal complement in the group of units of $\mathbb{Z} D_{16}^{+}$isomorphic to the group given by the following presentation:

\begin{tabular}{l|lllll} 
& {$\left[A_{0}, A_{1}\right]$} & $=R_{1} R_{2} R_{3} R_{0}$ & $=S_{3} S_{2} S_{1} S_{0}$ & $=$ \\
$A_{0}, A_{1}$, & $S_{3}^{-1} R_{2} S_{1}^{-1} R_{0}$ & $=S_{0}^{-1} R_{3} S_{2}^{-1} R_{1}$ & $=$ & & \\
$P_{0}, P_{1}, P_{2}, P_{3}$, & $P_{3}^{-1} A_{0}^{-1} Q_{2}^{-1} A_{0}^{-1} Q_{1} P_{0}$ & $=R_{0}^{-1} A_{1} Q_{0} S_{0}^{-1} P_{0}=S_{0}^{-1} Q_{0} R_{0}^{-1} A_{1} P_{0}=$ & $=$ \\
$Q_{0}, Q_{1}, Q_{2}, Q_{3}$, & $P_{0}^{-1} A_{1}^{-1} Q_{3}^{-1} A_{1}^{-1} Q_{2} P_{1}=R_{1}^{-1} A_{2} Q_{1} S_{1}^{-1} P_{1}=S_{1}^{-1} Q_{1} R_{1}^{-1} A_{2} P_{1}=$ & $=$ \\
$R_{0}, R_{1}, R_{2}, R_{3}$, & $P_{1}^{-1} A_{2}^{-1} Q_{0}^{-1} A_{2}^{-1} Q_{3} P_{2}=R_{2}^{-1} A_{3} Q_{2} S_{2}^{-1} P_{2}=S_{2}^{-1} Q_{2} R_{2}^{-1} A_{3} P_{2}=$ & $=$ \\
$S_{0}, S_{1}, S_{2}, S_{3}$ & $P_{2}^{-1} A_{3}^{-1} Q_{1}^{-1} A_{3}^{-1} Q_{0} P_{3}=R_{3}^{-1} A_{0} Q_{3} S_{3}^{-1} P_{3}=S_{3}^{-1} Q_{3} R_{3}^{-1} A_{0} P_{3}=1$. &
\end{tabular}

\section{REFERENCES}

[1] A. F. Beardon, The Geometry of Discrete Groups. Springer, 1983. MR85d:22026

[2] L. Bianchi, Sui gruppi de sostituzioni lineari con coeficienti appartenente a corpi quadratici imaginari, Math. Ann. 40 (1892), 332-412.

[3] J. Elstrodt, F. Grunewald and J. Mennicke, Groups Acting on Hyperbolic Space. Harmonic Annalysis and Number Theory, Springer, 1998. MF 98g:11058

[4] B. Fine, The Algebraic structure of the Bianchi Groups, Marcel Dekker, 1989. MR90h:20002

[5] L.R. Ford, Automorphic Functions (second edition), Chelsea, New York, 1951.

[6] R. Gow and B. Huppert, Degree problems of representation theory over arbitrary fields of characteristic 0. On Theorems of N. Itô and J.G. Thompson, J. Reine Angew. Math. MR:89b:20029

[7] E. Jespers and G. Leal, Describing units in integral group rings of some 2-groups, Comm. Algebra 19 (1991), 1809-1827. MR92f:20004

[8] E. Jespers and G. Leal, Degree 1 and 2 representations of nilpotent groups and applications to units of group rings, Manuscripta Math. 86 (1995), 479-498. MR96a:16030

[9] E. Jespers, G. Leal and Á. del Río, Products of free groups in the unit group of integral group rings, J. Algebra 180 (1996), 22-40. MR.96m:16045

[10] E. Jespers and M.M. Parmenter, Units of group rings of groups of order 16, Glasgow Math. J. 35 (1993), 367-379. MR95e:20009

[11] G. Leal and C. Polcino Milies, Isomorphic groups (and loop) algebras, J. Algebra 155 (1993), 195-210. MR.94a:16048

[12] B. Maskit, Kleinian groups, Springer-Verlag, 1988. MR90a:30132

[13] M.M. Parmenter, Free Torsion-free normal complements in integral group rings, Comm. Algebra 21 (10) (1993), 3611-3617. MR94k:16047

[14] V. Platonov and A. Rapinchuk, Algebraic groups and number theory, Academic Press, 1994. MR 95b:11039

[15] H. Poincaré, Mémoire sur les groupes kleinées, Acta Math. 3 (1883), 49-92.

[16] A. del Río and M. Ruiz, Computing large direct products of free groups in integral group rings, Comm. Algebra 30 (4) (2002), 1751-1767. MR2003a:20007

[17] S.K. Sehgal, Units of Integral Group rings, Longman Scientific and Technical Essex, 1993. MR $94 \mathrm{~m}: 16039$

[18] M.F. Vignéras, Aritmétique des algèbres de quaternions, Lect. Notes Math. 800, Springer, Berlin, Heidelberg, New York, 1980. MR 82i:12016

Departamento de Matemáticas, Universidad de Murcia, Campus de Espinardo, 30100 Murcia, Spain

E-mail address: antopita@um.es

Departamento de Matemáticas, Universidad de Murcia, Campus de Espinardo, 30100 Murcia, Spain

E-mail address: adelrio@fcu.um.es

Departamento de Métodos Cuantitativos e Informáticos, Universidad Politécnica de Cartagena, Paseo Alfonso XiII, 50, 30203 Cartagena, Spain

E-mail address: manuel.ruiz@upct.es 